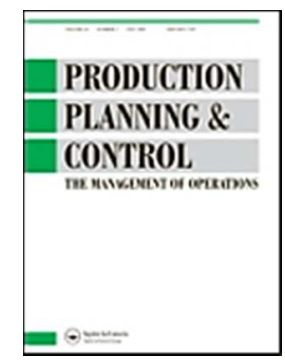

\title{
Opportunism in buyer-supplier exchange: a critical examination of the concept and its implications for theory and practice
}

\begin{tabular}{|r|l|}
\hline Journal: & Production Planning \& Control \\
\hline Manuscript ID & TPPC-2016-0121.R2 \\
\hline Manuscript Type: & Research \\
\hline Date Submitted by the Author: & 13-Apr-2018 \\
\hline Complete List of Authors: & $\begin{array}{l}\text { Kelly, Stephen; Edge Hill University, Business School } \\
\text { Wagner, Beverley; Strathclyde Business School } \\
\text { Ramsay, John; Staffordshire University }\end{array}$ \\
\hline Keywords: & Opportunism, Buyer-Supplier Exchange, Critique, Guile \\
\hline \multicolumn{2}{|l}{} \\
\hline
\end{tabular}

SCHOLARONE ${ }^{\text {m }}$

Manuscripts 


\title{
Opportunism in buyer-supplier exchange: a critical examination of the concept and its implications for theory and practice
}

\begin{abstract}
Claims that opportunism is widespread in the process of buyer-supplier exchange are commonplace, but direct supporting evidence for such claims is largely absent from the relevant literatures. This paper offers a critique of the treatment of opportunism in supply chains by re-establishing the importance of guile in the concept, and investigates existing published, empirical measures of buyer and supplier opportunistic behaviour. The paper offers evidence that, despite the frequency with which the concept is discussed in the literature and applied in research, and the emphasis given to the risks it generates for management, opportunism with guile between buyers and suppliers appears to be rare in practice. This paper is the first critical assessment of the concept's treatment in the Operations Management field, and it argues that practitioners are currently being poorly advised with respect to the phenomenon, as well as drawing conclusions for both practitioners and researchers that differ radically from the prevailing consensus on the subject.
\end{abstract}

\section{Keywords}

Opportunism, Guile, Buyer-Supplier Exchange, Critique 


\section{Opportunism in buyer-supplier exchange: a critical examination of the concept and its implications for theory and practice}

\section{Introduction}

Buyer-supplier relationships exist between firms to ensure that goods and services can be bought and sold in the most effective and efficient manners. Opportunism is deemed a behaviour that runs contrary to these aims and management devotes considerable time and resources in creating mechanisms to mitigate against it. The purpose of this article is to critically assess the current treatments of the concept of opportunism in relevant literatures by examining existing empirical evidence relating to the phenomenon and reflects the concerns that whilst opportunism is widely researched, it is rarely the central focus of much research and is consequently not completely understood (Hawkins et al. 2013). The specific focus of this article is an exploration of the role of opportunism with guile in buyer-supplier relationships, as this phenomenon is not explicitly considered by other theories or views and provides the contribution of this article.

The paper builds on research, which evaluates the presumption of the prevalence of opportunism in buyer-supplier exchanges (Hawkins et al, 2008) and questions the pervasive character of opportunism assumed by proponents of Transaction Economic theory (Moschandreas, 1997). Given the importance accorded to opportunism in business related research (Hawkins et al. 2013), we attempt to re-establish the significance of "guile" in Williamson's (1985) initial thesis of opportunism in his development of Transaction Cost Economics (TCE). In so doing the paper highlights methodological and survey design factors that may influence data gathering and interpretation of the phenomenon (Crosno and 
Dahlstrom, 2008). The practical aim of the paper is to ensure that management responses to the possible risks of opportunistic behaviour between buyers and suppliers match the magnitude of the threat the phenomenon poses. In the event of a mismatch, inappropriate management decisions may result. From an educational perspective and in line with Ketokivi and Mahoney (2016, p. 136), we suggest that "a thorough understanding can help us take TCE to the classroom in a constructive manner."

We argue that opportunism is central to TCE and widely used in the analysis of buyersupplier exchange relationship, although we acknowledge other theoretical perspectives could provide rival explanations of the phenomenon. The analysis that follows will be of particular value to researchers and practitioners with an interest in or responsibility for supply chain performance. The paper analyses the literature on opportunism from different academic fields to address three main questions:

- Is opportunism with guile common in buyer-supplier exchange?

- Are different forms of opportunism of equal importance to practitioners?

- Is opportunism a serious problem that demands significant management attention?

The current consensus in a variety of fields including Operations (OM), Marketing and Purchasing \& Supply Management (P\&SM) is that opportunism is a commonplace, significant threat to companies engaged in buyer-supplier exchange. However, this paper will show, through an evaluation of direct and indirect evidence, that the enactment of Williamson's (1985) original definition of opportunism with guile is not as common as the academic literature purports it to be. The contribution of this paper is threefold: 
I. A more critical treatment of opportunism, which more precisely defines the concept of guile and its role in opportunistic behaviour, allows us to more clearly differentiate between truly opportunistic behaviours and those that are overt in nature or are the results of mistakes, lack of knowledge and experience on the part of the supplier. Establishing the key role of guile therefore questions the pervasiveness of opportunism in buyer-supplier relationships.

II. This more precise view of guile means that practising purchasing managers can focus their managerial control activities on truly opportunistic behaviours and that extraordinary supplier evaluation and monitoring efforts are not required to provide protection from the effects of the opportunism. Practitioners can then more usefully devote these resources to more effective activities such as dealing with global and technological challenges resulting from an increasing reliance on digitalisation and the need to deal with more complex data.

III. Academic research, meanwhile, might usefully focus on both improving the construct validity of methods of measuring opportunism, and devising means of dealing with the epistemological difficulties of obtaining valid empirical responses, in order to arrive at a more accurate assessment of the actual incidence of the phenomenon. Further, the continued attribution of opportunism to more 'innocent' supplier behaviour results in the circular argument that opportunism is rife, which is inhibiting the development of trust and openness in buyer-supplier relationships.

The paper proceeds with an examination of the meaning of the term 'opportunism' in buyersupplier exchange, a review of the treatment of the phenomenon in a variety of literatures, and a critical analysis of the direct and indirect empirical evidence for the prevalence of buyer-supplier opportunism. This analysis results in the establishment of the lack of 
consideration of the role of guile in defining opportunistic behaviour and concludes with the finding that the phenomenon is not sufficiently common to warrant the currently recommended levels of management attention.

\section{The foundations of opportunism in the literature}

The concept of opportunism employed in OM stems from microeconomic theory, where it was introduced by Oliver Williamson to help explain the emergence of firms from pure market trading. Opportunism is an assumption at the heart of Transaction Cost Economics (TCE) and defined by Williamson as: ‘...self-interest seeking with guile' (Williamson, 1985, p.47). Because of its contribution to the understanding of the make-or-buy decision process in companies, the concept is of particular interest to the OM, P\&SM and Marketing fields where the largest number of published references is to be found. However, the term is employed widely in a variety of other fields: Organisation Science (Conner \& Prahalad, 1996), Finance (Douglas, 2007), Strategy (John, 2001), Human Resource Management (Lepak \& Snell, 1999). Close examination reveals that the usage of the term in the literature frequently does not match Williamson's (1985) original, idiosyncratic and deliberately narrow definition which he devised in order to explain the emergence of firms from the market. It will be argued that changes in the definition and flaws in the methods used to measure the concept have reduced the validity of empirical data, and practitioner advice derived from such studies may thus generate a misallocation of management resources.

The paper focuses on opportunism in buyer-supplier exchanges, and in particular analyses a small number of papers that offer direct and indirect evidence of the prevalence of buyer and supplier opportunism. Before the discussion narrows down to the empirical evidence of the 
frequency with which the phenomenon may be encountered, a brief overview summarising its treatment in the literatures from which those papers are drawn is offered to provide a context for that evidence. This section also establishes the key role that guile plays in opportunism and how its usage has developed from Williamson's original establishment of the concept.

\subsection{Definitions of opportunism}

The concept of opportunism refers to a feature of human behaviour rather than a technical, abstract economics concept, and the following discussion thus begins with general semantics and English usage. The dictionary definition of the word is relatively straightforward, opportunism is:

The art, policy, or practice of taking advantage of opportunities or circumstances often with little regard for principles or consequences.

(Merriam-Webster.com, 2014)

Or:

The policy of doing what is opportune or at the time expected...often used to imply sacrifice of principle or an undue spirit of accommodation to present circumstances.

(Oxford English Dictionary, 1989, p. 865)

The dictionary definition thus focuses on the taking of some kind of advantage involving a transgression against some principle, with an element of chance rather than deliberate planning. The definition devised by Williamson (1985) in Economics differs in several respects: 
The dictionary definition of 'guile' meanwhile is:

Insidious cunning, deceit, treachery.

(Oxford English Dictionary, 1989, p. 934)

Thus, in Williamson's (1985) version, the advantage being taken is self-interest and the main principles being violated (as his analysis indicates) are the business norms of being truthful and honouring contracts. Such relational norms are said to reduce opportunism as they are expectations about behaviour and govern exchange relationships between firms (Heide and John, 1992). The element of chance or lack of planning present in the dictionary definition is replaced with the opposite notion of deliberate planning or intent. Furthermore, because deliberately failing to honour a contract is very widely disapproved of in business, and dishonesty is met with opprobrium in both business and wider society, the perpetrators of this form of opportunism need to employ guile or deceit, and the behaviour is thus conducted in secret. When applied to buyer-supplier exchange the differences between the open or overt and secretive versions of the concept can be illustrated with reference to a buyer faced with a price increase from a supplier and this is shown in Table 1: 


\begin{tabular}{|l|l|l|}
\hline Concept & Behaviour & Illustrative example \\
\hline Overt opportunism & $\begin{array}{l}\text { The open pursuit of } \\
\text { advantage or benefit by one } \\
\text { party to an exchange at the } \\
\text { expense of their trading } \\
\text { opposite(s). }\end{array}$ & $\begin{array}{l}\text { The supplier forces a price } \\
\text { increase through without } \\
\text { offering any justification. }\end{array}$ \\
\hline $\begin{array}{l}\text { Opportunism-with- } \\
\text { guile }\end{array}$ & $\begin{array}{l}\text { The hidden pursuit of } \\
\text { advantage or benefit, with } \\
\text { the deliberate intent to } \\
\text { deceive, by one party to an } \\
\text { exchange at the expense of } \\
\text { their trading opposite(s). }\end{array}$ & $\begin{array}{l}\text { The supplier requests a } \\
\text { deliberately distorted data } \\
\text { in justification, and hides } \\
\text { the distortion. }\end{array}$ \\
\hline
\end{tabular}

Table 1 - Example of the overt and with-guile forms of opportunism (source - authors)

A supplier might employ Overt-opportunism if they were, for example, indifferent towards a buyer and did not care if they kept or lost their business. Suppliers keen to retain a buyer's business might also behave like this if they recognised that the buyer was so dependent on their output that they could demand whatever they liked without fear of losing the buyer's business.

\subsection{Current usage of opportunism - the effects of definition choice}

Firstly, we look at whether the concept of opportunism has been critically treated in the OM and Marketing literature, before going on to look at its empirical usage in sections 3.1 and 3.2. One might argue that in an OM paper, it is inappropriate to include data from Marketing studies. However, that field has made many more measurements of the phenomenon than any other to date, and their omission would have produced an indefensible narrowing of the analysis. Moreover, although they employ a different perspective, the Marketing studies examine the same phenomenon as those in OM, P\&SM and other fields. Many, use precisely 
the same measures in collecting data. Indeed, the concept of opportunism has been enthusiastically embraced by the two fields, as a search using the term "opportunism" in the Google Scholar and Summon search engines, and the EBSCO Business Premier and Emerald databases generated a list of likely target journals (see Table 2 below). These journals were considered to be the most relevant as taken from the Associated Business Schools (ABS) Journal Quality, and the search using “opportunism” generated a combined total of 1486 papers referring to the phenomenon.

\section{Operations Management}

Journal of Operations Management, International Journal of Operations and Production Management, Production, Planning and Control, Journal of Purchasing and Supply Management, Supply Chain Management an International Journal, Journal of Supply Chain Management, Journal of Production and Operations Management, Journal of Production Economics, International Journal of Physical Distribution and Logistics Management, Journal of Business logistics, International Journal of Logistics Management, Decision Sciences, European Journal of Operational Research

\section{Marketing}

Journal of Marketing Research, Journal of International Marketing, Psychology and Marketing, Journal of Business and Industrial Marketing, Journal of Retailing, Marketing Letters, Marketing Science, International Journal of Research in Marketing, Journal of the Academy of Marketing Science, Industrial Marketing Management, Journal of Marketing, European Journal of Marketing

Other managerial areas

Strategic Management Journal, Academy of Management Journal, Administrative Science Quarterly, British Journal of Management, Journal of Management

Table 2 - Relevant journals referring to the concept of opportunism

The papers were divided between the three researchers to scan for evidence of any critical and focused treatment of the concept of "opportunism" and this process identified 37 papers (as seen in Appendix A). Surprisingly, these contain only a few critical observations relating 
to the concept, as the focus is of these papers is on empirical measurement of the phenomenon. One early work (John, 1984) argued that, contrary to Williamson's claims: '...unrestrained self-interest maximization is not characteristic of human behaviour...' (1985, p. 278). The concept is widely referred to, and, in common with other fields, the dominant treatment is that of a problem requiring managing or minimizing, for example: Liu et al. (2010b, p. 844); Cavusgil et al. (2004, p. 8); Brown et al. (2000, p. 51). Thus Handley \& Benton (2012) comment on the challenges that have been made to Williamson's claim that opportunism is an innate human characteristic (Williamson, 1985, p. 56), and Gulbrandsen et al. (2009) deserve mention for referring to weaknesses in the original theory concerning the effects of opportunism and mechanisms for influencing it. In general, however, the concept has become so familiar that, with the exception of empirical papers employing the concept as a variable for measurement, references to it are usually made in passing, and the treatment is predominantly uncritical. The norm is for opportunism to be presented as a problem demanding management attention. For example, Cadden et al. (2015) show that opportunistic behaviour by the buyer (such as price renegotiation in situations of increased buying volumes) may result in reciprocal behaviour from the supplier (in the form of contract renegotiations in situations of decreasing buyer volumes) or that behaviour could be construed by one party as being opportunistic, e.g. in Chen et al. (2016) price increases from the supplier due to holidays were not believed by the buyer. Although such examples are nonetheless rigorous in the establishment of links between behaviour and interpretation and extend the scope of what may be considered opportunistic behaviour, introducing contextual matters into the discussion, they do not question the fundamental definition of opportunism used. 
Perhaps the most widely cited and hence influential, theoretical source that informs many of the later studies of the phenomenon in the OM and Marketing fields is Wathne \& Heide (2000) which has been cited in 374 publications (Scopus, accessed June 15, 2015, www.scopus.com). They begin with Williamson's 'self-interest seeking with guile' definition, and drawing on Masten (1988), describe it as "strong form" or "blatant" opportunism (Wathne \& Heide, 2000, p. 38). Given the importance of secrecy in Williamson's (1985) definition, the latter term is particularly inappropriate. They subdivide opportunism into two forms:

For example, opportunism in the form of quality shirking means that a party is withholding efforts, or passively failing to honor an agreement. In contrast, breaching a distribution contract by selling in an unauthorized territory involves an active effort. Wathne \& Heide, 2000, pp. 36-7 [emphasis in original]

Both of the examples they refer to may involve the deliberate use of deceit. They also draw on Macneil (1981) in the Law field, and employ his alternative definition of opportunism: "Self-interest seeking contrary to the principles of the relation in which it occurs." (Macneil, 1981, p. 1024). Unfortunately, their analysis loses clarity when they repeat a typing error in a footnote in Macneil's work (see Macneil, 1981, 1023), thus: “...a necessary starting point is a delineation of the term 'guile' which [Macneil] defines as “taking advantage of opportunities with little regard for principles or consequences." (Wathne \& Heide, 2000, p. 39). Those words, however, clearly derive from the Merriam-Webster definition (above) of opportunism not guile, and by transcribing this error they accidentally drop guile from their formal analysis and never clearly state their position on the behaviour. However, since some of the examples they subsequently discuss display guile and some do not, one may infer that 
Wathne \& Heide's (2000) treatment embraces both the with-guile and overt forms. Finally, whereas Williamson's (1985) theory focused narrowly on the violation of the principles of telling the truth and the honouring of contracts, Wathne \& Heide (2000) introduce a variety of other principles that may be violated including: 'the expectation of sharing benefits and burdens', 'restraints on unilateral use of power', 'general norms of equity', 'distributive justice', 'broad bargaining norms' and 'flexibility' which they define as the 'shared expectation that parties will adapt to changing circumstances' (p. 40).

It is self-evidently possible to devise any number of specialised definitions of the concept of opportunism. Williamson (1985) chose a very narrow, specialised form while Macneil (1981) and Wathne \& Heide (2000) favour a much broader concept. The relative merits of these alternative definitions of the concept is of no relevance to the current discussion, but the choice of definition made for research purposes is of importance because the breadth of the definition has a significant effect on the magnitude of the perceived risk or threat that opportunism poses in practice. The major differences between Williamson's (1985) and Wathne \& Heide (2000) and Macneil's (1981) definitions were the intent to deceive and hence the need for secrecy, and the number and nature of the principles being violated. In the comparison that follows the effects of those differences in practice is evaluated.

Neither Williamson's (1985) nor Wathne \& Heide (2000) and Macneil's (1981) definitions have any unambiguous implications for the possible costs generated by opportunism. They may be very high or very low in either case. However, when successfully deployed by a deceitful organisation, both the presence of opportunism-with-guile, and the source of its effects on the victim will be hard to detect, thus making it extremely difficult to manage and control. Wathne \& Heide (2000) and Macneil's (1981) definitions add openly opportunistic 
behaviours to those involving guile, but since these additional behaviours will be readily detected by victims, they will tend to be easier to manage.

Following criticisms of his opportunism assumption (see Donaldson, 1990 for example), Williamson repeatedly argued that his version of the phenomenon will be relatively uncommon in practice, see for example: (Williamson, 1998, p. 31; Williamson, 2005, p. 8; Williamson, 2008, p. 10, Williamson, 2009, pp. 150-151; Williamson, 2010, p. 678). His position is perhaps most clearly expressed here: [I do not believe]'... that most economic agents are engaged in opportunistic practices most of the time. Rather, most economic agents are engaged in business-as-usual, with little or no thought to opportunism, most of the time. Furthermore, Williamson repeatedly stressed that opportunism is only likely when the potential rewards for the perpetrator are very large or 'the stakes are high' - see for example (Williamson, 2008, p. 10; 2009, pp. 150-1; 2010, p. 678).

That opportunism does not continuously intrude is partly because many economic agents are well-socialized.' (Williamson, 1993, p. 98). Many people have been successfully persuaded by parents, schools and wider society that the behaviours of lying and cheating, upon which opportunism-with-guile relies, are undesirable. Moreover, those social norms are reinforced by a number of business norms connecting revealed deceit with damage to company reputation. The incidence of contract breach, for example, is low and companies rarely seek recourse to the law because: 'Two norms are widely accepted. (1) Commitments are to be honored in almost all situations; one does not welsh on a deal, (2) One ought to produce a good product and stand behind it.' (Macaulay, 1963, p. 63). 
Failure to conform to these norms will damage a company's reputation through the operation of 'governance mechanisms' such as the monitoring of the performance and behaviour of trading opposites. Companies who gain a reputation for being unreliable or deceitful may expect to lose customers or suppliers in the longer term. Klein discusses what he calls the 'self-enforcing range of contractual relationships', which is generated by the threat of the termination of trading relationships and damage to reputation in the marketplace (Klein 1996, p. 449). Discussing the building industry, Cox \& Thompson (1997) observe that: ‘... opportunism is curbed as the supplier is given the incentive of future work'. (p. 134) Fear of damage to company reputation tends to inhibit this form of bad behaviour.

Hence opportunism-with-guile may be rare in practice. In contrast, by not only adding Overt opportunism to Williamson's narrow definition but also increasing the range of principles to consider as targets for violation, Wathne \& Heide (2000) and Macneil's (1981) analysis would appear to open the door to a radical increase in the likely incidence of opportunism. Particularly since the high levels of disapproval that are associated with breach of contract and lying will not necessarily be generated by the violation of 'restraints on unilateral use of power', 'general norms of equity', 'broad bargaining norms' and the 'shared expectation that parties will adapt to changing circumstances' and so on.

Williamson $(1985 ; 1998)$ was not primarily interested in how buyers and suppliers interact with each other, his theory required behaviours that were so potentially threatening and destructive of trust in others that they would only be practiced in secret, and would compel individuals to stop trading with others and undertake the affected processes themselves, and thus explain the emergence of the firm from the market. In search of such an extreme phenomenon, his approach focuses on two principles whose violation is widely disapproved 
of and which need the perpetrators to act with guile as the consequences and costs to them if uncovered may be so severe that commercial survival is threatened. Such behaviour will be difficult to manage, but the incidence will be low. Wathne \& Heide (2000) and Macneil's (1981) approaches have no such limitations and their definition allows for a much less dramatic phenomenon whose effects can be so relatively innocuous that the perpetrators may display the behaviour openly without fear of significant reputation damage.

Hence in Wathne \& Heide (2000) and Macneil's (1981) treatment multiple principles may be involved, violation is regarded with differing levels of disapproval by business, guile may or may not be employed, the consequences and costs may be severe or slight, the behaviour may be difficult or relatively easy to manage, but the frequency should be markedly higher. There is no directly relevant data in the empirical literature on either the costs incurred by opportunistic behaviour in general or the difficulty of managing the phenomenon. It is possible, however, to obtain some data on the frequency of the phenomenon.

Unlike Williamson's theory (1985), Wathne \& Heide (2000) and Macneil's (1981) analysis can be used to predict that the opportunism should be relatively commonplace in practice. Hawkins et al, (2013, p. 905) state that "Because there is a dearth of empirical research examining the opportunism-performance relationship and because some of the literature on opportunism suggests that "opportunism pays" in certain situations", the paper turns now to that empirical evidence to assess the accuracy of that prediction. Having established that the notion of opportunism has moved from Williamson's (1985) initial treatment, i.e. with guile, and further established that there is a lack of critical treatment of the concept, we now turn to how empirical work has captured data on the phenomenon in using the broader understanding 
of opportunism. This has been perpetuated by the extensive use of Wathne \& Heide's (2000) work, which forms the basis of much empirical research on opportunism.

\section{Literature review methodology to identify empirical evidence of opportunism}

What follows is an assessment of the frequency with which opportunism occurs in buyersupplier exchange in order to more fully understand the use of guile in opportunism. This is a question of the empirical nature of a phenomenon that has been examined for more than a century: the behaviour of companies in supply chains (See for example Morris, 1910, p. 23) should not be seen as merely an arcane theoretical discussion about the microeconomic Theory of the Firm. After this length of time it should be possible to draw some conclusions about the extent and importance of the phenomenon. One meta-analysis observed that: '...much of the empirical literature rests on the general presumption of opportunism, but few studies measure opportunism directly. Given its theoretical centrality, it is surprising that the empirical literature is largely devoid of efforts to measure opportunism.' (Macher \& Richman, 2008, p. 40).

As shown in Section 2.2, a list of 1486 papers referring to the phenomenon were identified and in order to systematically review the literature on empirical evidence, we took the papers from the initial round of review (i.e. based on the search term "opportunism") and rather than looking for critical treatments (i.e. as per section 2), we looked at whether there had been any data collection method used to detect whether opportunism exists in buyer-supplier relationships. Firstly, we looked to see if any literature established whether "direct" evidence of opportunism existed. We looked at the papers generated from the journals in Table 2 to 
establish if there was any actual evidence and examples of opportunistic behaviour. The results and discussion of this are shown in section 3.1.

\subsection{Direct evidence}

The following section examines cases, that claim to describe the presence of opportunism, and attempts to match the phenomena described to the definitions above. The details from these cases are show in Appendix B and table 3 summarises the pertinent points. This table sets out the context of the studies, provides an indication of the possible opportunistic behaviour and also shows an alternative view that may equally explain the behaviour:

\begin{tabular}{|l|l|l|l|}
\hline Citation & Context & $\begin{array}{l}\text { Opportunistic } \\
\text { Behaviour }\end{array}$ & Alternative Explanation \\
\hline $\begin{array}{l}\text { Cox \& } \\
\text { Thompson } \\
(1997\end{array}$ & $\begin{array}{l}\text { Construction } \\
\text { industry }\end{array}$ & $\begin{array}{l}\text { Adversarial } \\
\text { relationships } \\
\text { encourage } \\
\text { opportunism: } \\
\text { Increasing annual } \\
\text { turnover through } \\
\text { contractual claims }\end{array}$ & $\begin{array}{l}\text { Many reasons why construction } \\
\text { companies present contractual } \\
\text { claims such as dealing with poor } \\
\text { management; inaccurate } \\
\text { forecasts; inaccurate information. }\end{array}$ \\
\hline $\begin{array}{l}\text { Anderson et } \\
\text { al. (2000) }\end{array}$ & $\begin{array}{l}\text { US car firms and die } \\
\text { manufacturers }\end{array}$ & $\begin{array}{l}\text { No evidence of buyer } \\
\text { opportunism, but } \\
\text { evidence of suppliers } \\
\text { causing delays that } \\
\text { could have been } \\
\text { attributed to } \\
\text { opportunism }\end{array}$ & $\begin{array}{l}\text { External suppliers do more work } \\
\text { to perfect the dies than internal } \\
\text { suppliers. This takes longer, } \\
\text { hence delays. }\end{array}$ \\
\hline $\begin{array}{l}\text { Wathne and } \\
\text { Heide (2000) }\end{array}$ & $\begin{array}{l}\text { Ford and supplier } \\
\text { Lear Corp. }\end{array}$ & $\begin{array}{l}\text { Supplier failed to } \\
\text { honour promises. }\end{array}$ & $\begin{array}{l}\text { Fords explanation of the } \\
\text { supplier's behaviour was that } \\
\text { rather than displaying } \\
\text { opportunism, Lear was suffering } \\
\text { from poor management. }\end{array}$ \\
\hline $\begin{array}{l}\text { Anderson } \\
\text { and Jap } \\
\text { (2005) }\end{array}$ & $\begin{array}{l}\text { Car firm and Parts } \\
\text { supplier. }\end{array}$ & $\begin{array}{l}\text { Supplier reduces the } \\
\text { number of coats of } \\
\text { paint to cut costs of } \\
\text { unit price. }\end{array}$ & $\begin{array}{l}\text { Evidence of Buyer using power } \\
\text { over supplier. Apparently } \\
\text { opportunistic behaviour is a result } \\
\text { of self-defence. }\end{array}$ \\
\hline $\begin{array}{l}\text { Oftrega and } \\
\text { quality and additional } \\
\text { services to new } \\
\text { customers at the same }\end{array}$ & $\begin{array}{l}\text { Internet } \\
\text { infrastructure } \\
\text { provider }\end{array}$ & $\begin{array}{l}\text { There is no market norm for } \\
\text { suppliers to tell buyers the prices } \\
\text { paid by all other customers. }\end{array}$ \\
\hline
\end{tabular}




\begin{tabular}{|c|c|c|c|}
\hline & & $\begin{array}{l}\text { price as the long term } \\
\text { customer. }\end{array}$ & \\
\hline $\begin{array}{l}\text { Mitrega and } \\
\text { Zolkiewski } \\
(2012)\end{array}$ & Coal supplier & $\begin{array}{l}\text { Coal supplier not } \\
\text { supplying coal at the } \\
\text { contracted price }\end{array}$ & $\begin{array}{l}\text { Close reading of the contract } \\
\text { showed that the supplier was } \\
\text { operating within the terms of the } \\
\text { contractual agreement. There } \\
\text { was no cheating or deliberate } \\
\text { distortion of information. }\end{array}$ \\
\hline $\begin{array}{l}\text { Cadden et al. } \\
(2015)\end{array}$ & Telecommunications & $\begin{array}{l}\text { The buying firm } \\
\text { threatened to use a } \\
\text { substitute from an } \\
\text { alternative supplier }\end{array}$ & $\begin{array}{l}\text { Traditional mechanism tactics in } \\
\text { renegotiations }\end{array}$ \\
\hline
\end{tabular}

Table 3 - Summary of direct evidence cases

Since the overwhelming majority of published research does not attempt to describe company behaviour in detail, and the cases above are the result of wide ranging searches of the literature, it is suggested that they represent a large sample of the relevant population. In summary, in the five papers discussed, three cases of 'opportunism' were ambiguous [Cox \& Thompson (1997); Anderson et al. (2000); Mitrega \& Zolkiewski (2012)]. One was ambiguous and may well have been a defensive supplier reaction to bad buyer behaviour (Wathne \& Heide, 2000), and one appeared to be competitive bargaining behaviour (Mitrega \& Zolkiewski (2012). Two appear to be examples of Overt-opportunism [Klein et al. (1978); Mitrega \& Zolkiewski (2012)]. There was only one clear example of opportunism-with-guile (Anderson \& Jap (2005), but the authors themselves suggest that the relevant case the behaviour may have been defensive in origin rather than unprompted aggressive pursuit of self-interest through deceit.

In conclusion, there is very little unambiguous, direct, empirical evidence that opportunism of any form constitutes a widespread, significant threat to company performance in buyersupplier exchanges. What little there is, however, is supplemented by a body of papers 
reporting the results of statistical investigations into the concept which offer indirect evidence of the presence and prevalence of the phenomenon, and this paper now turns to an examination of these.

\subsection{Indirect empirical evidence}

We then looked for "indirect" evidence of opportunism, i.e. where one of the parties believes that opportunistic behaviour may be affecting the buyer-supplier relationship.

By ignoring the normal focus on statistical evidence of associations between variables, and focusing instead on the data and the method of its collection, it is possible to extract an indirect measure of the incidence of opportunism from some empirical studies. For example, (Morgan et al. 2007) measured the phenomenon with a questionnaire asking respondents to consider a variety of statements relating to 'opportunistic' behaviours such as: ' ...considering this category of products, the supplier who has the most influence... [1]acks integrity when not closely monitored' (Morgan et al. 2007, p. 519). Respondents were asked to rate the accuracy of the statement using a Likert scale with: "..."strongly disagree" to "strongly agree" scale anchors.' (p. 520). Thus:

1 Strongly disagree

2 Disagree

3 Disagree somewhat

4 Neither agree nor disagree

5 Agree somewhat

6 Agree

7 Strongly agree 
In their descriptive statistics, they show a mean for opportunism of 2.98 (ibid). Because the mean is well below the neutral mid-point value of 4 on their scale, this suggests that when asked the extent to which they agreed with the relevant questions many of the respondents (buyers) selected the 'Strongly disagree', 'Disagree' or 'Disagree somewhat' choices on the questionnaire. Whilst it is possible that in any individual survey a large number of responses of 4 or above might be over-whelmed by a smaller number of very low responses, there is no reason to suppose that this particular pattern would appear on a regular basis. Consequently, if a large number of surveys generate means at or below the mid-point of the scale this would suggest that many respondents did not perceive the relevant behaviours to be a significant feature of buyer-supplier exchange. Thus, empirical studies focused on buyer-supplier exchange, employing a construct for opportunism, citing descriptive statistics including a mean for that construct, and describing the scale used to collect the data, can be used to generate an indirect measure of the prevalence of opportunism.

The details of the filtering process varied with the capabilities of the 'within journal' search option of the various publishers, but after removing those that did not contain the words "supplier", "mean" or "likert", a total of 345 papers remained that matched the specification above. These were read in detail to remove all studies that failed to refer to buyer-supplier exchange - thus some strategic partnership and alliance studies were excluded because interactions of this kind take many different forms only a few of which involve buyers working with suppliers, e.g. Das \& Teng, 2000, p. 13. Many alliance/partnership analyses do not focus on buyer-supplier exchanges, e.g.: Fan, 2011; Li, 2008; Luo, 2007; Johnson et al. 1996; Parkhe, 1993. Furthermore, some do not make clear what type of exchange process they are studying, see e.g. Delerue-Vidot, 2006. Non-empirical papers were removed, along 


\begin{tabular}{|c|c|c|c|c|c|c|c|}
\hline Source & Context & $\begin{array}{l}\text { Respond } \\
\text { ents }\end{array}$ & $\begin{array}{l}\text { Opp. mean } \\
\text { (Likert scale } \\
\text { format) }\end{array}$ & $\begin{array}{l}\text { Mean } \\
\text { at or } \\
\text { below } \\
\text { mid- } \\
\text { point } \\
\text { of scale } \\
\text { (freq.) }\end{array}$ & $\begin{array}{l}\text { Rank of } \\
\text { opp. mean } \\
\text { in study }\end{array}$ & $\begin{array}{l}\text { Opp. as } \\
\text { dependent } \\
\text { variable }\end{array}$ & $\begin{array}{l}\text { Self- } \\
\text { report } \\
\text { or } \\
\text { others }\end{array}$ \\
\hline & & & $\frac{\text { Supplier }}{\text { Opportunism }}$ & & & & \\
\hline $\begin{array}{l}\text { Handley } \\
\& \\
\text { Benton, } \\
2012\end{array}$ & $\begin{array}{l}\text { Large US } \\
\text { based } \\
\text { companies } \\
\text { with } \\
\text { domestic and } \\
\text { /or offshore } \\
\text { business } \\
\text { processes. }\end{array}$ & Suppliers & $\begin{array}{l}1.43,1.40, \\
1.33,1.75, \\
1.51,1.50, \\
1.29,1.26, \\
1.43 \\
\text { (7 point) }\end{array}$ & 9 & Lowest & $\mathrm{Y}$ & Self \\
\hline $\begin{array}{l}\text { Lui \& } \\
\text { Ngo, } \\
2012\end{array}$ & $\begin{array}{l}\text { Trading } \\
\text { companies in } \\
\text { garment and } \\
\text { toy industries } \\
\text { in Hong } \\
\text { Kong and } \\
\text { suppliers in } \\
\text { China. }\end{array}$ & Buyers & $\begin{array}{l}2.8 \\
(5 \text { point })\end{array}$ & 1 & Lowest & $\mathrm{N}$ & Others \\
\hline $\begin{array}{l}\text { Yang et } \\
\text { al. } 2011\end{array}$ & $\begin{array}{l}\text { Manufacturin } \\
\mathrm{g} \text { firms } \\
\text { covering a } \\
\text { wide range of } \\
\text { industries, } \\
\text { electronics, } \\
\text { computer } \\
\text { equipment, } \\
\text { chemicals, } \\
\text { apparel, }\end{array}$ & Buyers & $\begin{array}{l}2.46,3.62 \\
\text { (7 point) }\end{array}$ & 2 & Lowest & $\mathrm{N}$ & Others \\
\hline
\end{tabular}




\begin{tabular}{|c|c|c|c|c|c|c|c|}
\hline & $\begin{array}{l}\text { furniture, } \\
\text { food and } \\
\text { textiles in } \\
\text { China. }\end{array}$ & & & & & & \\
\hline $\begin{array}{l}\text { Yen \& } \\
\text { Barnes, } \\
2011\end{array}$ & $\begin{array}{l}\text { Anglo - } \\
\text { Taiwanese } \\
\text { buyer - seller } \\
\text { firms. }\end{array}$ & Buyers & $\begin{array}{l}3.07,2.96 \\
2.88,2.93 \\
(7 \text { point })\end{array}$ & 4 & Lowest & $\mathrm{Y}$ & Others \\
\hline $\begin{array}{l}\text { Samaha } \\
\text { et al. } \\
2011\end{array}$ & $\begin{array}{l}\text { Fortune } 500 \\
\text { firm (seller) } \\
\text { and its } \\
\text { resellers } \\
\text { (Channel } \\
\text { members). } \\
\text { Sectors } \\
\text { included } \\
\text { appliances, } \\
\text { automotive, } \\
\text { clothing, } \\
\text { electronics, } \\
\text { computers, } \\
\text { sport etc. }\end{array}$ & Buyers & $\begin{array}{l}2.23 \\
(5 \text { point })\end{array}$ & 1 & $\begin{array}{l}\text { Second } \\
\text { lowest }\end{array}$ & $\mathrm{N}$ & Others \\
\hline $\begin{array}{l}\text { Barthéle } \\
\text { my, } 2011\end{array}$ & $\begin{array}{l}\text { French } \\
\text { franchise } \\
\text { chains. }\end{array}$ & Buyers & $\begin{array}{l}3.49 \\
\text { (7 point) }\end{array}$ & 1 & Lowest & $\mathrm{N}$ & Others \\
\hline $\begin{array}{l}\text { Barnes et } \\
\text { al. } 2010\end{array}$ & $\begin{array}{l}\text { Western } \\
\text { exporters } \\
\text { from US, } \\
\text { Canada, GB, } \\
\text { Ireland, } \\
\text { Australia and } \\
\text { New Zealand } \\
\text { and } \\
\text { importing } \\
\text { firms based } \\
\text { in Hong } \\
\text { Kong. }\end{array}$ & Buyers & $\begin{array}{l}2.96,3.16 \\
3.00,3.28 \\
\text { (7 point) }\end{array}$ & 4 & $\begin{array}{l}\text { Second } \\
\text { lowest }\end{array}$ & $\mathrm{Y}$ & Others \\
\hline $\begin{array}{l}\text { Ghosh \& } \\
\text { John, } \\
2009\end{array}$ & $\begin{array}{l}\text { US } \\
\text { engineering } \\
\text { intensive } \\
\text { industry } \\
\text { sectors. }\end{array}$ & Buyers & $\begin{array}{l}3.13 \\
\text { (7 point) }\end{array}$ & 1 & $\begin{array}{l}\text { Second } \\
\text { lowest }\end{array}$ & $\mathrm{N}$ & Others \\
\hline $\begin{array}{l}\text { Lui et al. } \\
2009\end{array}$ & $\begin{array}{l}\text { Hong Kong } \\
\text { trading firms } \\
\text { and Chinese } \\
\text { suppliers. }\end{array}$ & Buyers & $\begin{array}{l}2.9 \\
(5 \text { point })\end{array}$ & 1 & $\begin{array}{l}\text { Equal } \\
\text { lowest }\end{array}$ & $\mathrm{N}$ & Others \\
\hline $\begin{array}{l}\text { Lado et } \\
\text { al. } 2008\end{array}$ & $\begin{array}{l}\text { US catalogue } \\
\text { intermediarie } \\
\text { s affiliated } \\
\text { with a large }\end{array}$ & Buyers & $\begin{array}{l}2.24 \\
(5 \text { point })\end{array}$ & 1 & Lowest & $\mathrm{N}$ & Others \\
\hline
\end{tabular}




\begin{tabular}{|c|c|c|c|c|c|c|c|}
\hline & retail firm. & & & & & & \\
\hline $\begin{array}{l}\text { Palmatie } \\
\text { r et al. } \\
2007\end{array}$ & $\begin{array}{l}\text { Business to } \\
\text { business } \\
\text { relationships } \\
\text { between a } \\
\text { major } \\
\text { Fortune 500 } \\
\text { company } \\
\text { (seller) and } \\
\text { its local } \\
\text { distributor } \\
\text { agents. } \\
\text { Businesses } \\
\text { cover } \\
\text { products } \\
\text { including } \\
\text { clothing, } \\
\text { hardware, } \\
\text { furniture and } \\
\text { appliances. }\end{array}$ & Buyers & $\begin{array}{l}2.06,2.13 \\
\text { (5 point) }\end{array}$ & 1 & $2^{\text {nd }}$ lowest & $\mathrm{N}$ & Others \\
\hline $\begin{array}{l}\text { Heide et } \\
\text { al. } 2007\end{array}$ & $\begin{array}{l}\text { Business to } \\
\text { Business } \\
\text { relationships } \\
\text { between } \\
\text { manufacturer } \\
\text { s (suppliers) } \\
\text { of building } \\
\text { materials } \\
\text { (doors, } \\
\text { windows, } \\
\text { frames, stairs, } \\
\text { roofing } \\
\text { products) and } \\
\text { their } \\
\text { downstream } \\
\text { buyers. }\end{array}$ & Suppliers & $\begin{array}{l}1.38,1.56 \\
\text { ( } 7 \text { point })\end{array}$ & 2 & \begin{tabular}{|l|} 
Lowest \\
\end{tabular} & $\mathrm{N}$ & Self \\
\hline $\begin{array}{l}\text { Morgan } \\
\text { et al. } \\
2007\end{array}$ & $\begin{array}{l}\text { UK } \\
\text { supermarket } \\
\text { retailers }\end{array}$ & Buyers & $\begin{array}{l}2.98 \\
\text { (7 point) }\end{array}$ & 1 & Lowest & $\mathrm{Y}$ & Others \\
\hline $\begin{array}{l}\text { Carson } \\
\text { et al. } \\
2006\end{array}$ & $\begin{array}{l}\text { Outsourced } \\
\text { R\&D } \\
\text { relationships } \\
\text { in new } \\
\text { product } \\
\text { development } \\
\text { in US }\end{array}$ & Buyers & $\begin{array}{l}\text { NB 13.81/28 } \\
\text { (responses } \\
\text { summed not } \\
\text { averaged) }\end{array}$ & 1 & Middle & $\mathrm{N}$ & Others \\
\hline $\begin{array}{l}\text { Wuyts \& } \\
\text { Geysken } \\
\text { s, } 2005\end{array}$ & $\begin{array}{l}\text { Small to } \\
\text { medium sized } \\
\text { firms in } \\
\text { Industrial and }\end{array}$ & Buyers & $\begin{array}{l}1.99 \\
\text { (7 point) }\end{array}$ & 1 & Lowest & $\mathrm{N}$ & Others \\
\hline
\end{tabular}




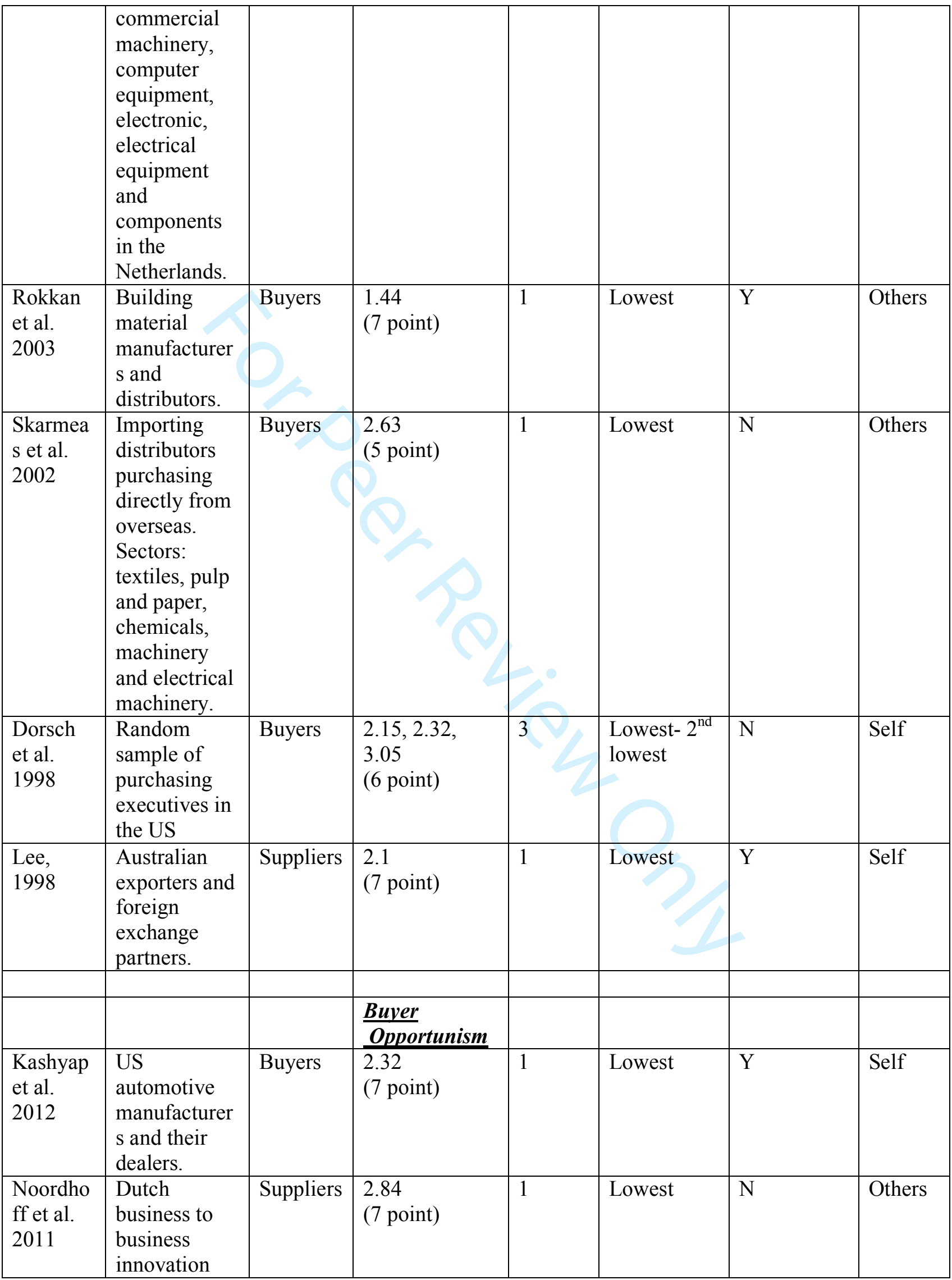




\begin{tabular}{|c|c|c|c|c|c|c|c|}
\hline & relationships. & & & & & & \\
\hline $\begin{array}{l}\text { Dev et } \\
\text { al. } 2011\end{array}$ & $\begin{array}{l}\text { Large hotel } \\
\text { firms in } \\
\text { North } \\
\text { America. }\end{array}$ & Buyers & $\begin{array}{l}1.99 \\
\text { (7 point) }\end{array}$ & 1 & Lowest & $\mathrm{Y}$ & Self \\
\hline $\begin{array}{l}\text { Ju et al. } \\
2011\end{array}$ & $\begin{array}{l}\text { Export } \\
\text { ventures in } \\
\text { China }\end{array}$ & Suppliers & $\begin{array}{l}2.42 \\
\text { (5 point) }\end{array}$ & 1 & Lowest & $\mathrm{Y}$ & Others \\
\hline $\begin{array}{l}\text { Liu et al. } \\
2010 b\end{array}$ & $\begin{array}{l}\text { Buyer- } \\
\text { supplier } \\
\text { relationships } \\
\text { in the context } \\
\text { of Chinese } \\
\text { household } \\
\text { appliances. }\end{array}$ & Suppliers & $\begin{array}{l}3.25 \\
\text { (7 point) }\end{array}$ & 1 & $\begin{array}{l}\text { Second } \\
\text { Lowest }\end{array}$ & $\mathrm{Y}$ & Others \\
\hline $\begin{array}{l}\text { Wong et } \\
\text { al. } 2005\end{array}$ & $\begin{array}{l}\text { Manufacturer } \\
\text { s and } \\
\text { suppliers } \\
\text { various } \\
\text { industries in } \\
\text { Shanghai. }\end{array}$ & Suppliers & $\begin{array}{l}2.62 \\
\text { (5 point) }\end{array}$ & 1 & $\begin{array}{l}\text { Second } \\
\text { Lowest }\end{array}$ & $\mathrm{Y}$ & Others \\
\hline $\begin{array}{l}\text { Cavusgil } \\
\text { et al. } \\
2004\end{array}$ & $\begin{array}{l}\text { US based } \\
\text { manufacturer } \\
\mathrm{s} \text { and } \\
\text { independent } \\
\text { foreign } \\
\text { distributers. }\end{array}$ & Suppliers & $\begin{array}{l}3.887,4.262, \\
4.543,4.585, \\
5.072 \\
(7 \text { point })\end{array}$ & 1 & Mixed & $\mathrm{Y}$ & Others \\
\hline $\begin{array}{l}\text { Jap, } \\
2003\end{array}$ & $\begin{array}{l}\text { Supply base } \\
\text { of a major } \\
\text { firm in the } \\
\text { automotive } \\
\text { industry } \\
\text { involved in } \\
\text { on-line } \\
\text { reverse } \\
\text { auctions. }\end{array}$ & Suppliers & $\begin{array}{l}2.94 \\
\text { (7 point) }\end{array}$ & 1 & Lowest & $\mathrm{Y}$ & Others \\
\hline $\begin{array}{l}\text { Brown et } \\
\text { al. } 2000\end{array}$ & $\begin{array}{l}\text { US Hotel } \\
\text { Industry. }\end{array}$ & Buyers & $\begin{array}{l}2.232 \\
\text { ( } 7 \text { point })\end{array}$ & 1 & Lowest & $\mathrm{Y}$ & Self \\
\hline $\begin{array}{l}\text { Dahlstro } \\
\text { m \& } \\
\text { Nygaard, } \\
1999\end{array}$ & $\begin{array}{l}\text { Norwegian } \\
\text { franchisee- } \\
\text { franchisor } \\
\text { relationships } \\
\text { in the oil } \\
\text { industry. }\end{array}$ & Buyers & $\begin{array}{l}3.06 \\
\text { (7 point) }\end{array}$ & 1 & Lowest & $\mathrm{N}$ & Others \\
\hline $\begin{array}{l}\text { Provan } \\
\& \\
\text { Skinner, } \\
1989\end{array}$ & $\begin{array}{l}\text { Farm and } \\
\text { Power } \\
\text { equipment } \\
\text { dealers and } \\
\text { primary } \\
\text { supplier }\end{array}$ & Buyers & $\begin{array}{l}2.52 \\
\text { (6 point) }\end{array}$ & 1 & 2nd lowest & $\mathrm{Y}$ & Self \\
\hline
\end{tabular}




\begin{tabular}{|c|c|c|c|c|c|c|c|}
\hline & $\begin{array}{l}\text { organisations } \\
\text { in US. }\end{array}$ & & & & & & \\
\hline & & & & & & & \\
\hline & & & $\begin{array}{l}\text { Both Buyer } \\
\text { and Supplier } \\
\text { Opportunism } \\
\end{array}$ & & & & \\
\hline $\begin{array}{l}\text { Caniëls } \\
\& \\
\text { Gelderm } \\
\text { an, } 2010\end{array}$ & $\begin{array}{l}\text { Information } \\
\text { and } \\
\text { communicati } \\
\text { on } \\
\text { Technology } \\
\text { professionals } \\
\text { in Dutch } \\
\text { local } \\
\text { government. }\end{array}$ & $\begin{array}{l}\text { Buyers } \\
\text { and } \\
\text { suppliers }\end{array}$ & $\begin{array}{l}\text { Suppliers: } 2.54 \\
\text { Buyers: } 2.03 \\
\text { (5 point) }\end{array}$ & 2 & $\begin{array}{l}\text { Second } \\
\text { Lowest } \\
\text { Lowest }\end{array}$ & $\mathrm{Y}$ & Both \\
\hline $\begin{array}{l}\text { Liu et al. } \\
2010 \mathrm{a}\end{array}$ & $\begin{array}{l}\text { Distributors } \\
\text { and } \\
\text { Manufacturer } \\
\text { s in Chinese } \\
\text { household } \\
\text { appliances. }\end{array}$ & $\begin{array}{l}\text { Buyers } \\
\text { and } \\
\text { suppliers }\end{array}$ & $\begin{array}{l}4.31 \\
\text { (7 point) }\end{array}$ & 0 & Lowest & $\mathrm{N}$ & Self \\
\hline $\begin{array}{l}\text { Liu et al. } \\
2009\end{array}$ & $\begin{array}{l}\text { Household } \\
\text { appliance } \\
\text { manufacturer } \\
\text { s and } \\
\text { distributors in } \\
\text { China. }\end{array}$ & $\begin{array}{l}\text { Buyers } \\
\text { and } \\
\text { suppliers }\end{array}$ & $\begin{array}{l}4.09 \\
\text { (7 point) }\end{array}$ & 0 & $\begin{array}{l}\text { Second } \\
\text { Lowest }\end{array}$ & $\mathrm{Y}$ & Others \\
\hline $\begin{array}{l}\text { Tokman } \\
\text { et al. } \\
2007\end{array}$ & $\begin{array}{l}\text { Greek SME, } \\
\text { 3PL } \\
\text { providers. }\end{array}$ & $\begin{array}{l}\text { Buyers } \\
\text { and } \\
\text { suppliers }\end{array}$ & $\begin{array}{l}3.08 \\
\text { (5 point) }\end{array}$ & 0 & $\begin{array}{l}\text { Second } \\
\text { lowest }\end{array}$ & $\mathrm{N}$ & Others \\
\hline $\begin{array}{l}\text { Kneyme } \\
\text { yer \& } \\
\text { Murphy, } \\
2005\end{array}$ & $\begin{array}{l}\text { Users and } \\
\text { Providers of } \\
\text { 3PL services } \\
\text { in US. }\end{array}$ & $\begin{array}{l}\text { Buyers } \\
\text { and } \\
\text { suppliers }\end{array}$ & $\begin{array}{l}\text { Suppliers: } 4.0 \\
\text { Buyers: } 3.1\end{array}$ & 1 & $\begin{array}{l}\text { Second and } \\
\text { third lowest }\end{array}$ & $\mathrm{N}$ & Others \\
\hline $\begin{array}{l}\text { Jap \& } \\
\text { Anderso } \\
n, 2003\end{array}$ & $\begin{array}{l}\text { Procurement } \\
\text { division of } 4 \\
\text { large } \\
\text { equipment } \\
\text { manufacturer } \\
\mathrm{s} \text { in } \\
\text { computing, } \\
\text { photography, } \\
\text { chemicals } \\
\text { and brewing. }\end{array}$ & $\begin{array}{l}\text { Buyers } \\
\text { and } \\
\text { suppliers }\end{array}$ & $\begin{array}{l}2.15 \\
\text { (7 point) }\end{array}$ & 1 & Lowest & $\mathrm{N}$ & Others \\
\hline $\begin{array}{l}\text { Jap, } \\
2001\end{array}$ & $\begin{array}{l}\text { Retailers and } \\
\text { manufactures } \\
\text { in chemical } \\
\text { industry. }\end{array}$ & $\begin{array}{l}\text { Buyers } \\
\text { and } \\
\text { suppliers }\end{array}$ & $\begin{array}{l}2.2 \\
\text { (7 point) }\end{array}$ & 1 & Lowest & $\mathrm{N}$ & Others \\
\hline
\end{tabular}




\section{Table 4 - Empirical results: Opportunism in Buyer-Supplier Exchange}

There are two clear outliers in table 4: Handley \& Benton, 2012, who generated extremely low means for opportunism, and Cavusgil et al. 2004 with unusually high means. The former authors used atypical measures of shirking and poaching behaviours (see further comments below). The latter created an atypical sample by deliberately selecting industrial sectors where they thought it likely that opportunism would be an unusually serious problem, and asking their respondents to focus on only their 'most challenging' trading experiences. The 'unusual' nature of both papers might justify their exclusion from what follows, however, since the results in the two papers in question, broadly speaking, cancel each other out, it was decided to leave them in. There are also a few papers with relatively high means in the 'both buyer and supplier' section, e.g.: Liu et al. 2009; 2010 and Tokman et al. 2007. The companies sampled in those papers include small and medium-sized enterprises (SMEs), Greek companies and some specifically featuring closely cooperative inter-company trading. This prompts speculation about whether opportunistic behaviour is perhaps related to size of firm, or is culture-specific, or is stimulated by especially close inter-company trading? However, with such a small number of papers, definitive statements are not possible at this time.

\subsection{Data interpretation}

The 37 empirical studies shown in Table 4 contain a total of 63 means for opportunism, of which $52(82.5 \%)$ were at or below the neutral mid-point of the relevant Likert scales used in the studies. Thus $82.5 \%$ of the papers appear to indicate that most respondents selected the 'Strongly disagree', 'Disagree' or 'Disagree somewhat' choices on the relevant 
questionnaires. Moreover, in more than a third (22) of the studies, the mean for opportunism was unambiguously the lowest recorded for all constructs measured using Likert scales. Thus, taken as a whole, the results in Table 4 above do not appear to support the claim that opportunism of any kind is widespread in buyer-supplier exchange. On the contrary, one might argue that this body of research appears to offer strong support for the view that, despite the conventional wisdom in the field, opportunistic behaviour is not commonplace between buyers and suppliers. This finding is reinforced by other scholars such as Moschandreas (1997) and Ketokvi and Mahoney (2016).

\section{Discussion}

\subsection{Extended the range of behaviours deemed opportunistic}

The 37 papers detailed above contained in Table 4 make use of a total of 185 relevant questions. Many of these were only slightly modified versions of the same idea, e.g.: 'Sometimes this supplier alters the facts slightly in order to get what they need.' (Lui \& Ngo, 2012, p. 93), and '[the supplier] Alters facts in order to meet their own goals and objectives.' (Samaha et al. 2011, p. 113). Others were re-worded to fit a specific market, e.g.: 'This supplier exaggerates needs to get what they desire.' (Skarmeas et al. 2002, p. 781) compared with: 'The contractor sometimes exaggerated the necessity of changes it wanted to the development plan or budget.' (Carson et al. 2006, p. 1076). It is evident that there is extensive and quite understandable item duplication resulting from the sound research design practice of using questions from previous surveys whose validity is assumed to have been established. In order to reduce the entire group to a more manageable size, a list was created 
and Table 5 shows the underlying, generic questions, the frequency with which each question was employed and the principles to whose breach the questions referred, thus:

\begin{tabular}{|c|c|c|}
\hline Question & Question (principles being violated in bold) & $\begin{array}{l}\text { Frequency } \\
\text { with which the } \\
\text { question has } \\
\text { been used } \\
\end{array}$ \\
\hline & The [trading opposite]: & \\
\hline & Honouring contracts/promises & \\
\hline 1 & sometimes promises to do things without actually doing them later. & 19 \\
\hline 2 & breaches formal or informal agreements to their benefit. & 15 \\
\hline 3 & does not always act in accordance with our contract(s). & 6 \\
\hline \multirow[t]{2}{*}{4} & $\begin{array}{l}\text { fails to deliver promises, as described in the contract, for its own } \\
\text { interests. }\end{array}$ & 1 \\
\hline & Truth Telling & \\
\hline 5 & alters the facts in order to get what they need. & 22 \\
\hline 6 & lies about certain things in order to protect its interests. & 19 \\
\hline 7 & exaggerates needs to get what they desire & 11 \\
\hline 8 & does not provide a completely truthful picture when negotiating & 9 \\
\hline 9 & has presented facts in such a way that has made them look good. & 6 \\
\hline 10 & $\begin{array}{l}\text { seems to believe that honesty does not pay when dealing with } \\
\text { partners }\end{array}$ & 4 \\
\hline 11 & makes false accusations & 3 \\
\hline \multirow[t]{2}{*}{12} & is candid with us (reverse scored) & 2 \\
\hline & Maintaining equity/distributive justice & \\
\hline 13 & $\begin{array}{l}\text { will try to take advantage of "holes" in our contract to further their } \\
\text { own interests. }\end{array}$ & 7 \\
\hline 14 & $\begin{array}{l}\text { feels it is OK to do anything within its means that will help further } \\
\text { its own interests. }\end{array}$ & 7 \\
\hline 15 & avoids fulfilling their responsibilities unless they are watched & 6 \\
\hline 16 & uses unexpected events to extract concessions from our firm. & 5 \\
\hline 17 & has benefited from our relationship to our detriment. & 4 \\
\hline 18 & withholds important information from us. & 4 \\
\hline 19 & fails to provide us with the support they are obliged to & 4 \\
\hline 20 & is unwilling to accept responsibility & 3 \\
\hline 21 & $\begin{array}{l}\text { expects my firm to pay for more than their fair share of the costs to } \\
\text { correct a problem }\end{array}$ & 2 \\
\hline 22 & $\begin{array}{l}\text { usually register a complaint if our company fails to meet our } \\
\text { cooperative agreements }\end{array}$ & 1 \\
\hline 23 & $\begin{array}{l}\text { expects to receive an unreasonably large share of the benefits from } \\
\text { our cooperative agreements }\end{array}$ & 1 \\
\hline 24 & tries to renegotiate contracts to its own advantage & 1 \\
\hline 25 & tends to escalate cost estimates as projects progress & 1 \\
\hline
\end{tabular}




\begin{tabular}{|c|c|c|}
\hline 26 & is less and less cooperative as projects progress & 1 \\
\hline 27 & $\begin{array}{l}\text { is reluctant to accept changes without receiving concessions and } \\
\text { compromises }\end{array}$ & 1 \\
\hline 28 & abuses displays of honesty on our part & 1 \\
\hline 29 & has coerced us unfairly in order to gain accessions & 1 \\
\hline 30 & is aloof toward us & 1 \\
\hline \multirow[t]{2}{*}{31} & fails to provide proper notification & 1 \\
\hline & Observing bargaining norms & \\
\hline \multirow[t]{3}{*}{32} & does not negotiate from a good faith bargaining perspective & 6 \\
\hline & Avoiding shirking & \\
\hline & $\begin{array}{l}\text { If [the customer] were not able to detect it, how likely are members } \\
\text { of our organization to... }\end{array}$ & \\
\hline 33 & $\begin{array}{l}\text { not assign your best people to your business or account with [the } \\
\text { customer]? }\end{array}$ & 1 \\
\hline 34 & provide a lower than agreed to level of resources? & 1 \\
\hline 35 & withhold information that may be beneficial to [the customer]? & 1 \\
\hline 36 & not share the benefits of process improvements? & 1 \\
\hline 37 & delay making agreed to investments in employee training? & 1 \\
\hline \multirow[t]{3}{*}{38} & delay making agreed to investments in new technology? & 1 \\
\hline & Avoiding poaching & \\
\hline & $\begin{array}{l}\text { If [the customer] were not able to detect it, how likely are members } \\
\text { of our organization to... }\end{array}$ & \\
\hline 39 & $\begin{array}{l}\text { use potentially proprietary information obtained through your } \\
\text { relationship with [the customer] to gain favor with other clients? }\end{array}$ & 1 \\
\hline 40 & $\begin{array}{l}\text { use potentially proprietary information obtained through your } \\
\text { relationship with [the customer] to help win business with other } \\
\text { customers? }\end{array}$ & 1 \\
\hline \multirow[t]{2}{*}{41} & $\begin{array}{l}\text { use potentially proprietary information obtained through your } \\
\text { relationship with [the customer] to develop new services that you } \\
\text { can offer in the marketplace? }\end{array}$ & 1 \\
\hline & Avoiding deceit & \\
\hline 42 & has tried to deceive us on several occasions & 1 \\
\hline
\end{tabular}

Table 5 - Generic questions in empirical surveys

Many of the behaviours described by that set of questions could only hope to have positive outcomes for the perpetrators if they possessed a power surplus over their trading partners and were prepared to use it to further their own interests, thus violating the principle of restraining from the 'unilateral use of power'. Question 27 addresses the 'shared expectation that parties will adapt to changing circumstances and question 32 refers directly to bargaining 
behaviour, albeit in a somewhat puzzling manner that is discussed below. Overall, one may conclude that the 37 empirical studies have incorporated, to varying degrees, all of the additional principles described by Wathne \& Heide (2000), and this is unlikely therefore to be a limiting factor on the recorded incidence of opportunism. The process of continually basing new research on existing survey constructs therefore serves to extend the perception of behaviours that can be considered opportunistic. As scholars use the term to apply to a wider range of behaviours, future research should focus on a return to Williamson's core definition.

\subsection{Distinguishing between motivations - re-establishing the key role of guile}

The critical factor defining the presence of all forms of opportunism is the motivation of the party breaking the promise. In studies where they are asked to describe the behaviour of their trading opposites (see final column in Table 4), the motivations underlying that behaviour are frequently unknown to the respondents. An examination of the questions reveals that they have differing abilities to capture data relating to, and subsequently distinguish between, not only Overt-opportunism and opportunism-with-guile, but also opportunistic and nonopportunistic behaviours in general. Consequently, versions of the questions above will, not only pick up incidences of opportunism with and without guile, but also other, nonopportunistic behaviours.

This lack of concern for the presence or absence of guile is shown in question 1: 'This supplier has sometimes promised to do things without actually doing them later.'(Ghosh \& John, 2009, p.603). This question appears in 19 papers with only minor variations in wording. Whilst the question appears superficially to match Williamson's (1985) definition, there are 
many reasons why companies fail to honour promises. Companies may do so as a result of both opportunism-with-guile or Overt-opportunism. However, such behaviour might equally be caused by unforeseeable events such as accidents, management incompetence, human error, working on the basis of inaccurate information such as forecasts, ambiguity in framing leading to differing interpretations of contracts and instructions and even offering wellintentioned but erroneous statements and so on. Similarly, all of the truth telling violation questions (5-12), with the exception perhaps of number 11, are capable of generating scores above the neutral mid-point on the scale in response to the various non-opportunistic reasons such as incompetence, accidents and the like. Reduced question validity of this kind will act to inflate the apparent incidence of opportunism.

To successfully capture the incidence of opportunism-with-guile, questionnaire items must exclude behaviours conducted openly. Failure to do so will exaggerate recorded frequencies. For example: 'this buyer will take advantage of 'holes' in our contracts to further their own interests' (Liu et al, 2010b, p. 849), or: 'The distributor has interpreted terms of the contract in [its] favour at our expense.' (Cavusgil et al. 2004, p. 17). If the distributor or buyer exhibits these behaviours openly without making any attempt to hide or disguise their actions, then this might be the result of Overt-opportunism or perhaps management incompetence and so on. Excluding the questions relating to the act of lying (which are dealt with below), only a few items in the studies were specifically intended to detect opportunism-with-guile by stressing the use of secrecy by the perpetrators. Thus (Morgan et al. 2007) include question 42 in Table 5: '...considering this category of products, the supplier who has the most influence has tried to deceive us on several occasions...' (p. 519). Six surveys including (Morgan et al. 2007) use variations on question 15: 'avoids fulfilling their responsibilities unless they are watched'. In each case these questions were mixed in with others less closely 
targeted on guile, thus making it impossible to single out the incidence of opportunism-withguile from those studies. Handley \& Benton (2010), in contrast, sought to capture the respondents' intent to deceive throughout their entire survey by preceding all questions with the phrase 'If [the customer] were not able to detect it, how likely are members of our organization to...'(questions 33-41). Their findings include the lowest means for opportunism in all of the studies in this analysis, and thus may support Williamson's claim that opportunism-with-guile will be unusual in practice. It should be noted however that another explanation for the very low incidence reported by Handley \& Benton is that respondents asked to self-report opportunistic behaviour are likely to under-report as a result of the effects of social acceptability bias. Few practitioners will be keen to tell researchers that they have behaved in a reprehensible manner. Thus, it would be reasonable to suppose that the real incidence of opportunism-with-guile in somewhat higher than that captured in Handley \& Benton (2010). The apparent incidence will also be boosted by above neutral responses to some of the questions that make no attempt to stress secrecy.

In addition to the distortions generated by social acceptability bias there is another severe epistemological difficulty in designing questionnaire items capable of detecting opportunismwith-guile. For example, one commonly employed question takes the form: 'This supplier breaches formal or informal agreements to their benefit'. If the supplier commits the breach openly then the behaviour may be Overt-opportunism. However, if the perpetrators use guile to hide their actions in order to avoid the disapproval that would follow their discovery, the victims will be unaware that any breach has occurred, unless the attempt is uncovered and thus fails in its objective. Thus, all such questions will, by definition, be unable to detect successful acts of opportunism-with-guile, and only capable of picking up both Overtopportunism and failed attempts at opportunism-with-guile. However, there will be no way of 
distinguishing between the overt and with-guile forms of behaviour in the published data. Where questions of this form are employed the true incidence of opportunism-with-guile may be higher than the results suggest, but unlike the incidence depressing effects of social acceptability bias, because the behaviour is detected, the combined incidence of opportunism with and without guile will not be artificially reduced.

Excluding the 16 questions specifically designed to detect secrecy and deceit and the 6 questions targeting negotiating practice (see below), all of the remaining questionnaire items are capable of generating positive, above neutral responses to, variously, failed opportunismwith-guile, Overt-opportunism, some routine negotiating practices and a variety of other nonopportunistic behaviours. Authors may have decided that it is either not necessary to search for evidence of guile, or perhaps too difficult to achieve that objective. Given the very high citation level of Wathne \& Heide's (2000) paper with its confusing handling of the concept of guile and emphasis on Overt-opportunism [53\% of the empirical papers discussed below that were published after 2000 cite Wathne \& Heide (2000) directly - see 'Comments' column in Appendix A: Definitions of opportunism in the empirical literature], belief that it is not necessary to search for guile is entirely understandable.

In Williamson's (1985) theory, the guile was needed to hide the reprehensible behaviour and would only be used when the potential benefits to the perpetrators were high enough to warrant the risk of reputation damage. Hence the incidence should be low. By ignoring guile the incidence should be increased as an extensive range of trivial, low cost/benefit behaviours will match the alternative, guile-free treatment of the phenomenon. It is, for example, not normal practice to attack your own organisation's image when talking to trading opposites. So buyers or suppliers might quite openly and casually present: 'facts in such a way that has 
made them look good.' (question 9) in minor events that have no significant monetary implications. Similarly, a supplier who explains a late delivery by reference to a vehicle breakdown might be perceived as using: 'unexpected events to extract concessions from our firm' (question 16), and so on. It is reasonable to suppose therefore that many of the remaining 162 questions will be likely to generate more above neutral responses, than questions designed to detect only high-costs behaviours carried out in secret.

\subsection{Accepted principles - negotiation practice}

As was discussed earlier, Williamson's theory focuses mainly on the principles of truthtelling and the honouring of contracts. One of Wathne \& Heide's (2000) contributions drawing on Macneil (1981), was to expand the number of principles that could be violated by opportunistic behaviour to include 'the expectation of sharing benefits and burdens', 'general norms of equity', 'distributive justice', 'restraints on unilateral use of power', the 'shared expectation that parties will adapt to changing circumstances' and 'broad bargaining norms'. (Wathne \& Heide's, 2000, p. 40). The first three of those principles are variations on the principle of behaving in a fair manner towards your trading opposites. These are comprehensively covered by questions 13-31 in Table 5 all of which relate to the principle of 'Maintaining equity/distributive justice'.

Wathne \& Heide (2000) refer to the principle of 'broad bargaining norms' and question 32: 'does not negotiate from a good faith bargaining perspective' clearly refers to this activity. It is possible, however that, the question might be misinterpreted by respondents unfamiliar with the detailed, American legal origins of the phrase. Particularly since there is one common form of negotiating that bears a superficial resemblance to opportunism. There are 
two main bargaining strategies employed in Buyer-supplier exchange - Distributive and Integrative (Lewicki et al. 2006). The former is competitive whereas the latter is entirely cooperative in nature and used, for example, in strategic partnerships. The majority of buyersupplier negotiations use a combination of tactics from both strategies. Distributive negotiation routinely involves the use of a number of tactics that may resemble opportunismwith-guile, including the deliberate distortion or withholding of information, lying, exaggerating, bluffing and the like.

These behaviours are regarded as perfectly acceptable within the context of the distributive negotiating process, but once negotiations are concluded and an agreement reached, the same behaviours revert to their 'unacceptable' status. In addition to question 32, 103 of the questions could return an above neutral response if the respondent was considering the behaviour of their trading partners during distributive negotiations. For example it is not uncommon for negotiators to either exaggerate a position (question 7) or refer to unexpected events to extract concessions (question 16) during a distributive negotiation. By accidentally recording non-opportunistic distributive negotiating behaviours as a form of opportunism, this reduced question validity is likely to create a false increase in the recorded incidence of the phenomenon.

\subsection{Summary of discussion}

There is likely to be some under-reporting of opportunism-with-guile, but Williamson (1985) has argued that this behaviour will be relatively unusual. Moreover, one may argue that this under-reporting will be more than compensated for by the over-reporting of apparent opportunism that will occur as many of the questions employed record above neutral 
responses to behaviours that have no opportunistic intent. The construct validity of many of the questions is open to question. However, the net effect of their reduced construct validity should be an over, rather than an under-reporting of the incidence of opportunism. Hence the conclusion that the very low incidence of opportunistic behaviours recorded is not the result of poorly designed questionnaire items. Indeed, the recorded incidence, despite being very low, may well be exaggerated. The published direct and indirect empirical evidence thus suggests that, contrary to the conventional wisdom in the relevant literatures, opportunistic behaviours of all kinds may indeed be relatively uncommon in buyer-supplier exchange (see also Moschandreas, 1979).

However, before one can conclude that management may safely ignore the concept, one objection remains. Although the incidence may be low, the effects of the phenomenon may be so severe that they cannot safely be ignored (Crosno and Dahlstron, 2008). In the Purchasing context, it would only take one major opportunistic act by a supplier of a critical product or service, performed at the wrong point in time, to cause a serious failure for a buyer's organisation. From a management perspective, what matters is the balance of the magnitude of the threat with the likelihood of its occurrence and not all instances of opportunism will have severe consequences. This follows the portfolio approach advocated initially by Kraljic (1983) in which categories of spend are differentiated according to their risk impact on operational disruption and also on bottom line profitability. This segmentation allows buying organizations to adopt different strategies to best deal with specific type of purchases. Companies aim to move their supply relationships to those of a less impactful nature (Gelderman and Van Weele, 2002) and have significantly reduced the number of major suppliers in recent years (Wagner \& Johnson, 2004), to try and establish closer relationships with fewer selected suppliers (e.g., Goffin et al., 1997). If a supplier should 
display such opportunistic behaviour, this will only be of significance if they also happen to supply a product or service that is both of strategic importance to the buyer, and difficult to re-source. However, the majority of any company's purchases are of not of strategic significance (e.g. 20\% of overall purchases as shown in Gangurde \& Chavan, 2016), and only a minority is difficult to re-source. Performance failures by most suppliers can be worked around with relatively little difficulty. Competent purchasing functions will already be paying close management attention to strategically important suppliers/purchases, regardless of the possible risk from opportunism. Once more, this reduces the likelihood and frequency of large-impact threats from this phenomenon. Similarly, if the overwhelming view of practitioners was that supplier relationships are inherent opportunistic, we would expect to see a decrease in the quantity and/or range of outsourcing activities, but the evidence is to the contrary (Financial Times).

The broader treatment of Wathne \& Heide (2000) not only makes opportunism much more common than the Williamson variant, but also much less commercially threatening. By rejecting Williamson's need for guile and increasing the range of principles that may be considered for potential violation, Wathne \& Heide (2000) and all the authors who follow this approach also remove the likelihood that the costs/benefits arising from the act of opportunism will be substantial. Indeed, taken to their logical limit, a principle such as their: 'shared expectation that parties will adapt to changing circumstances'

The definitions of opportunism as applied to Buyer-supplier exchange have been clarified to sharpen the effects of the presence or absence of guile on the frequency, difficulty of managing and potential associated costs generated by the phenomenon. Direct evidence of the presence of opportunism has been shown to be extremely limited and ambiguous in 
nature, and the indirect empirical evidence appears to contradict the claim that the opportunism is widespread in the process of buyer-supplier exchange.

\section{Implications}

\subsection{Research and methodological implications}

Clearly it would be unreasonable to criticise the empirical papers discussed above for a failure to test the underlying assumptions of the TCE model; their purpose is to search for statistical relationships between constructs and in some ways this reflects the perception that much of this research does not have opportunism as it's central focus (Hawkins et al. 2013) . Nevertheless, where research employs Likert scales in questionnaires that subsequently generate a mean for the dependent variable that is below the mid-way point on the scale (see the second last column in Table 4, one might suggest that alarm bells should begin to sound. This finding suggests that any positive correlations uncovered between the opportunism scale and independent variables indicate that increases in the various independent variables are associated with increases in the numerical value of the scale. However, if these variations are not sufficient to change the mean value of the scale to a value above the mid-point, then the variations only reduce the extent to which the respondents did not find their trading partners' behaviour to be opportunistic in nature. Thus, in such studies, findings of this kind suggest that the importance, if not the very existence of the core phenomenon being studied is under some doubt.

Moreover, the definition of opportunism offered by Macneil (1981) and Wathne \& Heide (2000) has so little in common with the deliberately narrow, specialised version devised by Williamson (1985) that work on buyer-supplier relationships should ensure that it is clear 
which "type" of opportunism is being used. Our research suggests that there is lack of critique relating "opportunism with guile" as Williamson meant. The word "guile" has been lost and the way surveys are designed perpetuates this view.

It is recommended therefore that there is an urgent need to answer, instead the following questions:

- How common-place are the different forms of opportunism?

- How do the risks and threats posed by the different forms to victims and perpetrators compare?

This will require significant improvements in the construct validity of measures of opportunism and solutions to the epistemological difficulties discussed above that confound the task of measuring the incidence of the phenomenon. This research highlights the weaknesses of previous methodologies, particularly due to the social desirability effect (as per Crosno \& Dahlstrom, 2008) when trying to uncover "opportunism with guile" in buyersupplier exchanges and also the difficulty in obtaining direct empirical evidence when there are potential disparities between reasons and actions/behaviour. Given that most of the previous research on the topic have been based on surveys it seems appropriate to consider qualitative methods such as observation or critical incident techniques to explore these relationships more closely, over a period of time and to gain a deeper understanding of the phenomenon.

\subsection{Management implications}

From a management perspective, there is a risk that an uncritical acceptance of the supposed prevalence of opportunism will encourage organisations to adopt a variety of policies and 
behaviours inimical to their long-term financial wellbeing. If buyers assume that suppliers are essentially untrustworthy in nature, they might, for example, develop an unnecessarily adversarial trading attitude that would prevent the potential benefits that flow from more cooperative inter-company trading. They might also be reluctant to make long-term commitments that would enhance the process of exchange with suppliers. Similarly, suppliers wary of opportunistic buyers might hesitate before sharing information capable of improving the buyer's effectiveness. Suspicious buyers may devote excessive time and effort to the construction of unnecessarily complex contractual arrangements, or perhaps the waste of resources on expensive policies intended to police supplier quality control procedures, and so on. However, the results of the analysis above suggest that in the interests of attaining maximum system efficiency, management should avoid devoting large amounts of resources to the control of opportunism.

\section{Conclusions and contributions}

It is now possible to offer answers to the questions the paper set out to tackle:

- Is opportunistic behaviour common in buyer-supplier exchange?

- Are different forms of opportunism of equal importance to practitioners?

○ Is opportunism a serious problem that demands significant management attention?

We make no comment on the issue with regard to staff relations within organisations (e.g. Head \& Lucas, 2004) or between organisations in other forms of inter-company relations such as strategic alliances (e.g. Das, 2004). Nevertheless, TCE theory predicts that high levels 
of opportunism-with-guile will be unusual in markets (Williamson, 1985), and the direct and indirect evidence discussed above indicates that opportunism, both with guile and without, is a relatively rare phenomenon in buyer-supplier exchange. It is not suggested that opportunism poses no risk, but although Overt-opportunism displayed by very powerful buyers may be relatively common in some markets, managements are familiar with these challenges and need little help from academia in identifying or coping with them. Logic suggests that forms of opportunism that pose a high risk to the would-be perpetrators will occur very infrequently, whereas those with a low risk such as Overt-opportunism with limited commercial implications will be more common-place.

Overall, we suggest that there is a possibility that opportunism will occasionally generate serious problems for a very small number of companies. However, the frequency of the occurrence of severe problems will be so low that it does not warrant the allocation of significant management resources beyond those routinely allocated to strategically critical customers or suppliers. Therefore, these can be seen as being serious events whose occurrence is so unusual that they are not regarded as being worthy of management attention.

Finally, a persuasive piece of evidence that the risk is very small lies in the absence of records of cases of opportunistic behaviour causing catastrophic survival problems for companies. It is reasonable to suppose that such events would have made some impression and been cited, yet they appear to be absent from the relevant literatures. We would suggest that opportunism falls into the category of phenomena that are real and with potentially serious consequences, but also sufficiently rare as to require little dedicated management attention. 
The available empirical evidence on many aspects of the phenomenon is very limited and this prompts some recommendations for future research. The current research on opportunism is towards further exploration of methods of controlling or minimising occurrences of opportunism. However, our contribution calls for the relevance of "guile" to be reestablished and our detailed review of the empirical evidence shows that it may be an unusual, relatively low-risk phenomenon and suggests this current approach is not well founded. From this perspective there has been a move away from the original Williamson definition to cover behaviours, which we class as overt opportunism, which can be uncovered and controlled by behavioural means, openness and trust in the buyer-supplier exchange relationships. Even with overt opportunism, our research indicates that this is still not significant. Our contribution calls for the relevance of "guile" to be re-established and to go back to the source of the concept rather than creating an academic consensus through circular use of ill- defined surveys.

\section{References}

Anderson, S., Glenn, D. and Sedatole, K. 2000. "Sourcing Parts of Complex Products: Evidence on Transactions Costs, High-Powered Incentives and Ex-Post Opportunism.” Accounting, Organizations and Society 25 (8): 723-749.

Anderson, E. and Jap, S. 2005. "The Dark Side of Close Relationships", MIT Sloan Management Review, Spring, pp. 75-82.

Barnes, B., Leonidou, L., Siu, N. and Leonidou, C. 2010. “Opportunism as the Inhibiting Trigger for Developing Long-Term-Oriented Western Exporter-Hong Kong Importer Relationships.” Journal of International Marketing. 18(2): 35-63. 
Barthélemy, J. 2011. “Agency and Institutional Influences on Franchising Decisions." Journal of Business Venturing. 26 (1):93-103.

Brown, J., Dev, C. and Lee, D. 2000. "Managing Marketing Channel Opportunism: The Efficacy of Alternative Governance Mechanisms.” Journal of Marketing. 64 (2): 51- 65.

Cadden, T., Marshall, D., Humphreys, P. and Yang, Y. 2015. "Old Habits Die Hard: Exploring The Effect of Supply Chain Dependency and Culture on Performance Outcomes and Relationship Satisfaction.” Production Planning \& Control. 26 (1): 53-77.

Caniëls, M., and C. Gelderman. 2010. "The Safeguarding Effect of Governance Mechanisms in Inter-Firm Exchange: The Decisive Role of Mutual Opportunism.” British Journal of Management 21 (1): 239-254.

Carson, S., A. Madhok, and T. Wu, 2006. "Uncertainty, Opportunism, and Governance: The Effects of Volatility and Ambiguity on Formal and Relational Contracting." Academy of Management Journal 49 (5):1058-1077.

Cavusgil, S., S. Deligonul, and C. Zhang, 2004. "Curbing Foreign Distributor Opportunism: An Examination of Trust, Contracts, and the Legal Environment in International Channel Relationships.” Journal of International Marketing 12(2):7-27.

Chen, J., A. Sohal, and D. Prajogo, 2016. "Supply Risk Mitigation: A Multi-Theoretical Perspective.” Production Planning \& Control 27 (10):53-863.

Conner, K., and C. Prahalad, 1996. "Resource-Based Theory of the Firm: Knowledge Versus Opportunism." Organization Science 7 (5):477-501.

Cox, A., and I. Thompson, 1997. "Fit For Purpose' Contractual Relations: Determining a Theoretical Framework for Construction Projects." European Journal of Purchasing and Supply Management 3 (3):127-135.

Crosno, J. L., and R. Dahlstrom, 2008. "A Meta-Analytic Review of Opportunism In Exchange Relationships.” Journal of the Academy of Marketing Science 36:191-201. 
Dahlstrom, R., and A. Nygaard, 1999. “An Empirical Investigation of Ex Post Transaction Costs in Franchised Distribution Channels.” Journal of Marketing Research 36(2):160-170.

Das, T., and B. Teng, 2000. “A Resource-Based Theory of Strategic Alliances.” Journal of Management 26 (1): 31-61.

Das, T. (2004) "Time $\square$ span and risk of partner opportunism in strategic alliances.” Journal of Managerial Psychology 19 (8): 744-759.

Delerue-Vidot, H. 2006. "Opportunism and Unilateral Commitment: The Moderating Effect of Relational Capital." Management Decision 44 (6):737-751.

Deligonul, S., D. Kim, A. Roath, and E. Cavusgil, 2006. "The Achilles' Heel of an Enduring Relationship: Appropriation of Rents Between A Manufacturer and Its Foreign Distributor.” Journal of Business Research 59 (7): 802-810.

Dev, C., S. Grzeskowiak, and J. Brown, 2011. “Opportunism in Brand Partnerships: Effects of Coercion and Relationship Norms." Cornell Hospitality Quarterly 52(4): 377-387.

Donaldson, L. 1990. "The Ethereal Hand: Organizational Economics and Management Theory." Academy of Management Review 15 (3): 369-381.

Dorsch, M., S. Swanson, and S. Kelley, 1998. "The Role of Relationship Quality in the Stratification of Vendors as Perceived by Customers." Journal of The Academy of Marketing Science 26 (2):128-142.

Douglas, A. 2007. "Managerial Opportunism and Proportional Corporate Payout Policies." Managerial Finance 33 (1): 26-42.

Fan, Y. 2011. “Influence of Parent Company's Organizational Dimensions on Its JV Performances - Opportunism Occurrence and Control Efficacy." International Journal of Business and Management 6 (7): 51-57.

Financial Times. 2013. The unstoppable rise of outsourcing.

https://www.ft.com/content/ee63a82c-d353-11e2-b3ff-00144feab7de. 
Gelderman, C.J., Van Weele, A.J., 2002. Strategic direction through purchasing portfolio management: a case study. Journal of Supply Chain Management 38 (2), 30-37.

Ghosh, M. and G. John 2009. "When Should Original Equipment Manufacturers Use Branded Component Contracts with Suppliers?.” Journal of Marketing Research 46 (5): 597611.

Goffin, K., Szwejczewski, M., \& New, C. (1997). Managing suppliers: When fewer can mean more. International Journal of Physical Distribution and Logistics Management 27(7): 422 436.

Gulbrandsen, B., K. Sandvik, and S. Haugland, 2009. “Antecedents of Vertical Integration: Transaction Cost Economics and Resource-Based Explanations." Journal of Purchasing and Supply Management 15 (2): 89-102.

Handley, S., and W. Benton, 2012. "The Influence of Exchange Hazards and Power on Opportunism in Outsourcing Relationships.” Journal of Operations Management 30 (1-2): 55-68.

Hawkins, T., T. Pohlen, and V. Prybutok, 2013. "Buyer Opportunism in Business-toBusiness Exchange.” Industrial Marketing Management 42 (8): 1266-1278.

Head, J., R. Lucas, (2004) "Employee relations in the non $\square$ union hotel industry: a case of “determined opportunism”?" Personnel Review 33 (6):.693-710.

Heide, J.B., and G. John, 1992. "Do Norms Matter in Marketing Relationships?.” The Journal of Marketing. 56: 32-44.

Heide, J. B. 1994. "Interorganizational Governance in Marketing Channels." The Journal of Marketing 58:71-85.

Heide, J., K. Wathne, and A. Rokkan, 2007. "Interfirm Monitoring, Social Contracts, and Relationship Outcomes.” Journal of Marketing Research 44 (3): 425-433. 
Jap, S. 2003. "An Exploratory Study of the Introduction of Online Reverse Auctions." Journal of Marketing 67 (3) 96-107.

Jap, S., and E. Anderson, 2003. "Safeguarding Interorganizational Performance and Continuity Under Ex Post Opportunism.” Management Science 49(12): 1684-1701.

Jap, S. 2001. "Perspectives on Joint Competitive Advantages in Buyer-Supplier Relationships.” International Journal of Research in Marketing 18 (1-2): 19-35.

John, G. 1984. “An Empirical Investigation of Some Antecedents of Opportunism in a Marketing Channel.” Journal of Marketing Research 21(August): 278-89.

John, D. 2001. "Strategy and Opportunism in European Retail Internationalization.” British Journal of Management 12(4): 253-266.

Johnson, J., J. Cullen, and T. Sakano, 1996. "Opportunistic Tendencies in IJVs with the Japanese: The Effects of Culture, Shared Decision Making, and Relationship Age." The International Executive 38 (1):79-94.

Ju, M., J. Murray, M. Kotabe, and G. Gao, 2011. "Reducing Distributor Opportunism in The Export Market: Effects of Monitoring Mechanisms, Norm-Based Information Exchange, and Market Orientation.” Journal of World Business 46 (4): 487-496.

Kashyap, V., K., Antia, and G. Frazier, 2012. “Contracts, Extracontractual Incentives, and Ex Post Behavior in Franchise Channel Relationships." Journal of Marketing Research 49 (2):260-276.

Ketokivi, M., and J.T. Mahoney, 2016. "Transaction Cost Economics as a Constructive Stakeholder Theory, Academy of Management Learning 15(1): 123-138.

Klein, B. 1996. "Why Hold-Ups Occur: The Self-Enforcing Range of Contractual Relationships.” Economic Enquiry 34(3): 444-463.

Klein, B., R. Crawford, and A. Alchian, 1978. "Vertical Integration, Appropriable Rents, and the Competitive Contracting." Journal of Law and Economics 21(2): 297-32. 
Knemeyer, A.M., P.R. Murphy, 2005. "Is the Glass Half Full Or Half Empty? An Examination of User and Provider Perspectives Towards Third-Party Logistics Relationships." International Journal of Physical Distribution \& Logistics Management 35 (10): 708-727.

Kraljic, P., 1983. Purchasing must become supply management. Harvard Business Review 61 (5): 109-117.

Lado, A., R. Dant, and A. Tekleab, 2008. "Trust-Opportunism Paradox, Relationalism, and Performance in Interfirm Relationships: Evidence from the Retail Industry." Strategic Management Journal 29 (4):401-423.

Lee, D.J. 1998. "Developing International Strategic Alliances Between Exporters and Importers: The Case of Australian Exporters." International Journal of Research in Marketing 15( 4):335-348.

Lepak, D., and S. Snell, 1999. "The Human Resource Architecture: Toward a Theory of Human Capital Allocation and Development." Academy of Management Review 24 (1):3148.

Lewicki, R., B. Saunders, and J. Minton, 2006. Negotiation. McGraw-Hill, New York, NY. Li, N. 2008 "Religion, Opportunism, and International Market Entry Via Non-Equity Alliances or Joint Ventures."Journal of Business Ethics 80 (4): 771-789.

Liu, Y., Y. Luo, and T. Liu, 2009. “Governing Buyer-Supplier Relationships Through Transactional and Relational Mechanisms: Evidence from China.” Journal of Operations Management 27( 4):294-309.

Liu, Y., Y. Li, and J. Xue, 2010a. "Transfer of Market Knowledge in a Channel Relationship: Impacts of Attitudinal Commitment and Satisfaction." Industrial Marketing Management 39 (2):229-239. 
Liu, Y., S. Chenting, L. Yuan, and L. Ting, 2010b. "Managing Opportunism in a Developing Inter Firm Relationship: The Interrelationship of Calculative and Loyalty Commitment." Industrial Marketing Management 39 (5): 844-852.

Lui, S., Y-Y Wong, and W. Liu, 2009. “Asset Specificity Roles in Interfirm Cooperation: Reducing Opportunistic Behavior or Increasing Cooperative Behavior?.”Journal of Business Research 62 (11):1214-1219.

Lui, S., and H-Y. Ngo, 2012. "Drivers and Outcomes of Long-Term Orientation in Cooperative Relationships.” British Journal of Management 23 (1): 80-95.

Luo, Y. 2007. “Are Joint Venture Partners More Opportunistic in a More Volatile Environment?." Strategic Management Journal 28 (1): 39-60.

Macaulay, S. 1963. "Non-Contractual Relations in Business. A Preliminary Study." American Sociological Review 28 (1):55-67.

Macher, J., and B. Richman, 2008. "Transaction Cost Economics: An Assessment of Empirical Research in the Social Sciences.” Business and Politics 10 (1): 1-63.

Macneil, 1. R. 1981. "Economic Analysis of Contractual Relations: its Shortfalls and the Need for a 'Rich Classificatory Apparatus." Northwestern University Law Review 75 February: 1018-1063.

Masten, S. E. 1988. "Equity, Opportunism, and the Design of Contractual Relations." Journal of Institutional and Theoretical Economics 144 (1):180-95.

Merriam-Webster dictionary. 2014. Available at: http://www. Merriam-Webster.com (accessed: 9 June 2014).

Mitrega. M., and J. Zolkiewski, 2012. "Negative Consequences of Deep Relationships With Suppliers: an Exploratory Study in Poland.” Industrial Marketing Management 41 (5): 886894. 
Morgan, N., A. Kaleka, and R. Gooner, 2007. "Focal Supplier Opportunism in Supermarket Retailer Category Management.” Journal of Operations Management 25 (2) 512-527.

Morris, R. 1910. Railroad Administration, D. Appleton and Company, New York and London.

Moschandreas, M. 2002. “The Role of Opportunism in Transaction Cost Economics, Journal of Economic Issues 31 (1): 39-58.

Noordhoff, C., K., Kyriakopoulos, C., Moorman, P. Pauwels, and B. Dellaert, 2011. "The Bright Side and Dark Side of Embedded Ties in Business-to-Business Innovation.” Journal of Marketing 75 (5): 34-52.

Oxford English Dictionary (1989), Second Edition, Vol. 6, Clarendon Press, Oxford.

Palmatier, R., R. Dant, and D. Grewal, 2007. “A Comparative Longitudinal Analysis of Theoretical Perspectives of Interorganizational Relationship Performance.” Journal of Marketing 71 (4)172-194.

Parkhe, A. 1993. "Strategic Alliance Structuring: A Game Theoretic and Transaction Cost Examination of Interfirm Cooperation.” Academy of Management Journal 36(40): 794-829.

Provan, K. and S. Skinner, 1989. "Interorganizational Dependence and Control as Predictors of Opportunism in Dealer-Supplier Relations.” Academy of Management Journal 32 (1): 202212.

Rokkan, A., J. Heide, and K. Wathne, 2003. "Specific Investments in Marketing Relationships: Expropriation and Bonding Effects.” Journal of Marketing Research 40 (2): $210-224$

Samaha, S., Palmatier, R. and Dant, R. 2011. "Poisoning Relationships: Perceived Unfairness in Channels of Distribution.” Journal of Marketing 75(3): 99-117.

Sanjaykumar R. Chavan, A, 2016. "Benchmarking of purchasing practices using Kraljic approach", Benchmarking: An International Journal 23 (7): 1751-1779. 
Skarmeas, D., C. Katsikeas, and B. Schlegelmilch, 2002. "Drivers of Commitment and Its Impact on Performance in Cross-Cultural Buyer-Seller Relationships: The Importer's Perspective.” Journal of International Business Studies 33 (4): 757-783.

Tokman, M., R., Richey, L. Marino, and K. Weaver, 2007. "Exploration, Exploitation and Satisfaction in Supply Chain Portfolio Strategy.” Journal of Business Logistics. 28 (1): 25-56. Wagner, S., \& Johnson, J. 2004. Configuring and Managing Strategic Supplier Portfolios. Industrial Marketing Management, 33(8), 717-730.

Walton, M. 1997. “When Your Partner Fails You.” Fortune 135 (10): 151-154.

Wang, X., and Z. Yang, 2013. "Inter-Firm Opportunism: A Meta-Analytic Review and Assessment of its Antecedents and Effect on Performance." Journal of Business \& Industrial Marketing 28(2):137 - 146 .

Wathne, K. and J. Heide, 2000. “Opportunism in Interfirm Relationships: Forms, Outcomes and Solutions.” Journal of Marketing 64( 4): 36-51.

Williamson, O. 1985. The Economic Institutions of Capitalism: Firms, Markets, Relational Contracting. The Free Press, New York, NY.

Williamson, O. 1993. "Opportunism and its Critics", Managerial and Decision Economics 14 (2): 97-107.

Williamson, O. 1998. "Transaction Cost Economics: How It Works; Where It Is Headed”, Economist 146 (1): 23-58.

Williamson, O. 2005. "The Economics of Governance." The American Economic Review 85 (2): $1-18$.

Williamson, O. 2008. "Outsourcing: Transaction Cost Economics and Supply Chain Management.” The Journal of Supply Chain Management 44 (2): 5-16.

Williamson, O. 2009. "Pragmatic Methodology: A Sketch, With Applications to Transaction Cost Economics." The Journal of Economic Methodology 16 (2): 145 - 157. 


\begin{abstract}
Williamson, O. 2010. "Transaction Cost Economics: The Natural Progression." The American Economic Review 100 (3): 673-690.

Wong, A., D., Tjosvold, and Z-Y. Yu, 2005. "Organizational Partnerships in China: SelfInterest, Goal Interdependence, and Opportunism." Journal of Applied Psychology 90 (4): 782-791.
\end{abstract}

Wuyts, S. and I. Geyskens, 2005: "The Formation of Buyer-Supplier Relationships: Detailed Contract Drafting and Close Partner Selection.” Journal of Marketing. 69 (4): 103-117.

Yang, Z., C. Zhou, and L. Jiang, 2011. "When Do Formal Control and Trust Matter? A Context-Based Analysis of the Effects on Marketing Channel Relationships in China." Industrial Marketing Management 40 (1): 86-96.

Yen, D., and B. Barnes, 2011. “Analyzing Stage and Duration of Anglo-Chinese BusinessTo-Business Relationships.” Industrial Marketing Management 40 (3):346-357. 


\begin{tabular}{|c|c|c|}
\hline Literature Source & Definition of Opportunism & Comments \\
\hline $\begin{array}{l}\text { Handley and } \\
\text { Benton, } 2012\end{array}$ & $\begin{array}{l}\text { 'Opportunism is defined as } \\
\text { "self-interest seeking with } \\
\text { guile" (Williamson, 1975, p. } \\
\text { 6). The behavioral assumption } \\
\text { of opportunism in TCT } \\
\text { presupposes that if given the } \\
\text { opportunity, individuals will } \\
\text { naturally act in a deceitful, } \\
\text { self-serving manner.' p. } 56\end{array}$ & $\begin{array}{l}\text { Williamson's } 1975 \text { definition is used. } \\
\text { Guile and/or deceit present. Wathne \& } \\
\text { Heide (2000) cited. }\end{array}$ \\
\hline Lui \& Ngo, 2012 & $\begin{array}{l}\text { 'Transaction cost economics } \\
\text { assumes the exchange partners } \\
\text { to be opportunistic, and will } \\
\text { seek self-interest at the expense } \\
\text { of the common interests of a } \\
\text { relationship (Williamson, } \\
\text { 1985).' p. } 81\end{array}$ & $\begin{array}{l}\text { Williamson's } 1985 \text { reference, but guile } \\
\text { omitted. Wathne \& Heide (2000) } \\
\text { cited. }\end{array}$ \\
\hline Kashyap et al. 2012 & Does not define & Wathne \& Heide (2000) cited \\
\hline Yang et al. 2011 & $\begin{array}{l}\text { '...opportunism, or "self- } \\
\text { interest seeking with guile," } \\
\text { which includes such behaviors } \\
\text { as lying and cheating, as well } \\
\text { as more subtle forms of deceit, } \\
\text { like violating agreements } \\
\text { (Williamson, 1985: p.47).' p. } \\
90\end{array}$ & $\begin{array}{l}\text { Williamson's } 1985 \text { definition is used. } \\
\text { Guile and/or deceit present. Wathne \& } \\
\text { Heide (2000) not cited. }\end{array}$ \\
\hline $\begin{array}{l}\text { Noordhoff et al. } \\
2011\end{array}$ & Does not define & Wathne \& Heide (2000) cited. \\
\hline Ju et al. 2011 & $\begin{array}{l}\text { '...franchisees may be tempted } \\
\text { to engage in opportunistic } \\
\text { behavior by "wilfully } \\
\text { disregarding the franchisor's } \\
\text { goals in pursuit of their own } \\
\text { entrepreneurial interests"...' } \\
\text { p. } 95\end{array}$ & $\begin{array}{l}\text { Guile omitted. Wathne \& Heide } \\
\text { (2000) cited. }\end{array}$ \\
\hline Yen \& Barnes, 2011 & $\begin{array}{l}\text { 'From a buyer's perspective, } \\
\text { opportunistic behavior occurs } \\
\text { when the seller takes } \\
\text { unexpected action for its own } \\
\text { gain but generates negative } \\
\text { outcomes for the relationship } \\
\text { partner...' p. } 349\end{array}$ & $\begin{array}{l}\text { Guile omitted. Wathne \& Heide } \\
\text { (2000) not cited. }\end{array}$ \\
\hline Samaha et al. 2011 & $\begin{array}{l}\text { "We use the term "seller } \\
\text { opportunism" to capture the } \\
\text { channel member's perception } \\
\text { of the degree to which the }\end{array}$ & $\begin{array}{l}\text { Williamson's } 1975 \text { definition is used. } \\
\text { Guile and/or deceit present. Wathne \& } \\
\text { Heide (2000) not cited. }\end{array}$ \\
\hline
\end{tabular}




\begin{tabular}{|c|c|c|}
\hline & $\begin{array}{l}\text { seller engages in self-interest- } \\
\text { seeking behaviors with guile.' } \\
\text { p. } 102\end{array}$ & \\
\hline Barthélemy, 2011 & $\begin{array}{l}\text { '...franchisees may be tempted } \\
\text { to engage in opportunistic } \\
\text { behavior by "wilfully } \\
\text { disregarding the franchisor's } \\
\text { goals in pursuit of their own } \\
\text { entrepreneurial interests" ...'p. } \\
95\end{array}$ & $\begin{array}{l}\text { Guile omitted. Wathne \& Heide } \\
\text { (2000) not cited. }\end{array}$ \\
\hline Dev et al. 2011 & $\begin{array}{l}\text { '...possibility arises that one } \\
\text { partner or the other will } \\
\text { work deceptively to its own } \\
\text { advantage.' p. } 377\end{array}$ & $\begin{array}{l}\text { Deceit present. Wathne \& Heide } \\
\text { (2000) cited. }\end{array}$ \\
\hline $\begin{array}{l}\text { Caniëls \& } \\
\text { Gelderman, } 2010\end{array}$ & $\begin{array}{l}\text { '...'self interest seeking with } \\
\text { guile' (Williamson, 1975, p. 6). } \\
\text { Opportunism includes all kinds } \\
\text { of deceitful behaviour .' p. } 239\end{array}$ & $\begin{array}{l}\text { Williamson's } 1975 \text { definition is used. } \\
\text { Guile and/or deceit present. Wathne \& } \\
\text { Heide (2000) not cited. }\end{array}$ \\
\hline Barnes et al. 2010 & $\begin{array}{l}\text { 'In brief, opportunism reflects } \\
\text { a form of behavior in which } \\
\text { some element of deceit is } \\
\text { apparent...' p. } 35\end{array}$ & $\begin{array}{l}\text { Deceit present. Wathne \& Heide } \\
\text { (2000) cited. }\end{array}$ \\
\hline Liu et al. (2010)a & $\begin{array}{l}\text { Williamson's } 1985 \text { definition is } \\
\text { used. }\end{array}$ & $\begin{array}{l}\text { Williamson's } 1985 \text { definition is used. } \\
\text { Guile and/or deceit present. Wathne \& } \\
\text { Heide (2000) not cited. }\end{array}$ \\
\hline Liu et al. (2010)b & $\begin{array}{l}\text { 'Opportunism, defined as the } \\
\text { extent to which a channel } \\
\text { member perceives his partner } \\
\text { to engage in "self-seeking } \\
\text { behaviors with guile" } \\
\text { (Williamson, 1975)... 'p.846 }\end{array}$ & $\begin{array}{l}\text { Williamson's } 1975 \text { definition is used. } \\
\text { Guile present. Wathne \& Heide (2000) } \\
\text { not cited. }\end{array}$ \\
\hline Ghosh \& John, 2009 & $\begin{array}{l}\text { '...by self-interested strategic } \\
\text { behavior and guile on the part } \\
\text { of the supplier during contract } \\
\text { execution.' p. } 602\end{array}$ & $\begin{array}{l}\text { Guile present. Wathne \& Heide (2000) } \\
\text { not cited. }\end{array}$ \\
\hline Liu et al. 2009 & $\begin{array}{l}\text { 'Opportunism is defined by } \\
\text { Williamson as self-interest } \\
\text { seeking with guile (1985).', p. } \\
294\end{array}$ & $\begin{array}{l}\text { Williamson's } 1985 \text { definition is used. } \\
\text { Guile present. Wathne \& Heide (2000) } \\
\text { cited. }\end{array}$ \\
\hline Lui et al. 2009 & $\begin{array}{l}\text { '... self-interest seeking with } \\
\text { guile.' p. } 1215\end{array}$ & $\begin{array}{l}\text { Williamson's } 1975 \text { definition is used. } \\
\text { Guile present. Wathne \& Heide ( } 2000) \\
\text { not cited. }\end{array}$ \\
\hline Lado et al. 2008 & $\begin{array}{l}\text { '...opportunism refers to } \\
\text { 'calculated efforts [by an } \\
\text { exchange agent] to mislead, } \\
\text { distort, disguise, obfuscate, or } \\
\text { otherwise confuse' } \\
\text { (Williamson, 1985: 47) an } \\
\text { exchange party.' p. } 403\end{array}$ & $\begin{array}{l}\text { Williamson's } 1985 \text { definition is used. } \\
\text { Guile present. } \\
\text { Wathne \& Heide (2000) not cited. }\end{array}$ \\
\hline Palmatier et al. 2007 & Does not define & Wathne \& Heide (2000) cited. \\
\hline
\end{tabular}




\begin{tabular}{|c|c|c|}
\hline Heide et al. 2007 & $\begin{array}{l}\text { 'Supplier opportunism. The } \\
\text { scale describes the extent to } \\
\text { which the supplier engages in } \\
\text { "self-interest seeking with } \\
\text { guile" (see Williamson's [1975, } \\
\text { p. 6]' p. } 429\end{array}$ & $\begin{array}{l}\text { Williamson's } 1975 \text { definition is used. } \\
\text { Guile present. Wathne \& Heide (2000) } \\
\text { not cited. }\end{array}$ \\
\hline Tokman et al. 2007 & Does not define & Wathne \& Heide (2000) not cited. \\
\hline Morgan et al. 2007 & $\begin{array}{l}\text { '...is more likely to engage in } \\
\text { guileful self-interest seeking, } \\
\text { i.e., opportunistic behavior ...', } \\
\text { p. } 515\end{array}$ & $\begin{array}{l}\text { Williamson definition with guile. } \\
\text { Wathne \& Heide (2000) cited. }\end{array}$ \\
\hline Carson et al. 2006 & $\begin{array}{l}\text { 'Williamson (1985) defined } \\
\text { opportunism as self-interest } \\
\text { eking of a strategic (i.e., } \\
\text { secretive, deceptive, or } \\
\text { guileful) nature undertaken to } \\
\text { redirect profits from vulnerable } \\
\text { partners.', p. } 1059\end{array}$ & $\begin{array}{l}\text { Williamson's } 1985 \text { definition is used. } \\
\text { Guile present. Wathne \& Heide (2000) } \\
\text { not cited. }\end{array}$ \\
\hline $\begin{array}{l}\text { Wuyts \& Geyskens, } \\
2005\end{array}$ & $\begin{array}{l}\text { 'Opportunism is defined as } \\
\text { self-interest seeking with } \\
\text { guile...' p.106 }\end{array}$ & $\begin{array}{l}\text { Williamson definition. Guile present. } \\
\text { Wathne \& Heide (2000) not cited. }\end{array}$ \\
\hline Wong et al. 2005 & $\begin{array}{l}\text { 'The possibility of } \\
\text { opportunistic behavior is an } \\
\text { important barrier to } \\
\text { collaboration because it raises } \\
\text { fears of exploitation as partners } \\
\text { pursue their self-interests with } \\
\text { guile (Williamson, 1985, } \\
\text { 1991).' p782 }\end{array}$ & $\begin{array}{l}\text { Williamson's } 1985 \text { definition is used. } \\
\text { Guile present. Wathne \& Heide (2000) } \\
\text { cited. }\end{array}$ \\
\hline $\begin{array}{l}\text { Kneymeyer \& } \\
\text { Murphy, } 2005\end{array}$ & $\begin{array}{l}\text { '...deceit-oriented violation of } \\
\text { implicit or explicit promises } \\
\text { about one's appropriate or } \\
\text { required role behavior.' p. } 712\end{array}$ & $\begin{array}{l}\text { Guile present. Wathne \& Heide (2000) } \\
\text { not cited. }\end{array}$ \\
\hline Cavusgil et al. 2004 & $\begin{array}{l}\text { '... it is possible to have a } \\
\text { relationship that is } \\
\text { characterized as cooperative in } \\
\text { an aggregate sense but that } \\
\text { over time may offer } \\
\text { opportunities for self-interest } \\
\text { seeking at the other party's } \\
\text { expense...' p. } 211\end{array}$ & $\begin{array}{l}\text { Guile omitted. Wathne \& Heide } \\
\text { (2000) cited. }\end{array}$ \\
\hline Jap, 2003 & $\begin{array}{l}\text { '...defined as self-interest- } \\
\text { seeking with guile.', p. } 98\end{array}$ & $\begin{array}{l}\text { Guile present. Wathne \& Heide (2000) } \\
\text { cited. }\end{array}$ \\
\hline $\begin{array}{l}\text { Jap \& Anderson, } \\
2003\end{array}$ & $\begin{array}{l}\text { 'Opportunism is self-interest } \\
\text { seeking with guile', p. } 1686\end{array}$ & $\begin{array}{l}\text { Guile present. Wathne \& Heide } \\
\text { (2000) cited. }\end{array}$ \\
\hline Rokkan et al. 2003 & Not defined. & Wathne \& Heide (2000) cited. \\
\hline Skarmeas et al. 2002 & $\begin{array}{l}\text { Williamson's } 1985 \text { definition is } \\
\text { used. }\end{array}$ & $\begin{array}{l}\text { Guile present. Wathne \& Heide (2000) } \\
\text { cited. }\end{array}$ \\
\hline Jap, 2001 & $\begin{array}{l}\text { 'self-interest seeking with } \\
\text { guile,', p. } 24\end{array}$ & $\begin{array}{l}\text { Guile present. Wathne \& Heide (2000) } \\
\text { cited. }\end{array}$ \\
\hline
\end{tabular}




\begin{tabular}{|l|l|l|}
\hline Brown et al. 2000 & $\begin{array}{l}\text { Williamson's 1975 definition is } \\
\text { used. }\end{array}$ & Guile present. \\
\hline $\begin{array}{l}\text { Dahlstrom \& } \\
\text { Nygaard, 1999 }\end{array}$ & $\begin{array}{l}\text { Williamson's 1985 definition is } \\
\text { used. } \\
\text { a.calculated efforts to mislead } \\
\text { and confuse trading partners . } \\
\text { p. 161 }\end{array}$ & Guile present. \\
\hline Dorsch et al. 1998 & $\begin{array}{l}\text { Williamson's 1975 definition is } \\
\text { used. }\end{array}$ & Guile present. \\
\hline Lee, 1998 & $\begin{array}{l}\text { Williamson's 1975 definition is } \\
\text { used. }\end{array}$ & Guile present. \\
\hline $\begin{array}{l}\text { Provan \& Skinner, } \\
1989\end{array}$ & $\begin{array}{l}\text { Williamson's 1975 definition is } \\
\text { used. }\end{array}$ & Guile present. \\
\hline
\end{tabular}




\section{Appendix B - Direct evidence cases (further details)}

Cox \& Thompson (1997) suggest that the 'peculiar' nature of the UK construction industry has created: '...adversarial arms-length relations with the parties selecting opportunistic behaviour rather than working together.' (p. 129). They claim 'considerable' evidence thus: '...a recent survey of the top 50 UK contractors revealed that a quarter earned between $10 \%$ and $15 \%$ of their annual turnovers through contractual claims with clients or suppliers, and a further third earned between $5 \%$ and $10 \%$ of their turnovers in a similar way...' ibid. There are a large number of reasons why construction companies resort to contractual claims such as responding to incompetent management practices, dealing with inaccurate forecasts/information and so on. Opportunism of any kind is only one of them. In the absence of evidence that opportunism is the main cause, the incidence of opportunism in this market remains indeterminate. Anderson et al. (2000) studied relations between US car firms and die manufacturers. They found no evidence of buyer opportunism, but did find evidence of suppliers causing delivery delays that may have been attributable to opportunism. However, these effects were explained by the supplier as the result of: '... anticipating that their parts are likely to be blamed for problems, external suppliers do more work to perfect the dies (relative to part-level specifications) than internal suppliers resulting in "better" parts (lower rework) but taking longer to achieve (longer submission delays). (Anderson et al. 2000, 745). Since it impossible to judge the veracity of the supplier's claims, it is not possible to arrive at an unequivocal identification of the form of company behaviour described.

In the same year, Wathne \& Heide (2000) described two relevant cases. One features Ford and a supplier (Lear Corp.), who failed to honour promises. However, the gap between the supplier's promises and actions was so wide that the chances of guile remaining undetected 
were minimal. If it had been uncovered, it would have damaged Lear's relations with Ford. Consequently, it is most unlikely that that they set out to deliberately deceive Ford - for details see Walton, (1997). This may therefore have been a case of Overt-opportunism. However, a Ford cost analyst commenting on one aspect of Lear's behaviour is quoted saying: "They're not venal, just incompetent" (Walton, 1997, 154). It is possible that, rather than displaying opportunism, Lear may simply have suffered from poor management. Once more a definitive identification of opportunism is impossible. Moreover, even if we tentatively conclude that it was an example of Overt-opportunism, the description of Ford's behaviour at the time includes the following phrases: 'Ford seemed wedded to old smashmouth tactics'; the program manager was a '...tough-minded Ford veteran with an especially combative attitude toward suppliers' and so on (Walton, 1997). Given the combination of Ford's power and aggressive attitude, it is possible that some of Lear's behaviour may have been defensive reaction to earlier attacks from Ford. Companies who act 'opportunistically' in response to previous bad behaviour on the part of trading partners may well be breaching the principles of telling the truth or honouring contracts, however they are not simply 'taking advantage' of their trading opposites. They may be acting to avoid the breach of a different, conflicting principle such as Wathne \& Heide's 'general norms of equity', 'restraints on unilateral use of power', the need to avoid 'exploiting vulnerability' and so on. An unambiguous identification of transgressive, opportunistic behaviour on the part of one trading partner is impossible when multiple conflicting principles are present and under attack. In such cases, it is necessary to examine the behaviour of all the parties involved, and the sequence of events. Only the first 'bad' behaviour in circumstances such as those just described would be opportunistic in the sense of one party 'taking advantage' of the other, but details of precisely who did what and when are rarely available in such cases. It is possible, therefore, that Lear's behaviour in the above case was not something arising in the 
market beyond the buyer's control that required management attention. Ford may have caused the behaviour to arise in the first place, and if so, the 'problem' might have been avoided if the buyer had behaved more reasonably.

In Anderson \& Jap (2005), a supplier dealing with a car company is quoted stating: 'If one decides to eliminate the first three coats of paint put on the component, well, then one cuts costs on the price of the unit, and that profit will be shared with Purchasing, which will recuperate a piece of the pie. Now, if one doesn't say anything, all the savings are ours... [emphasis in the original]' (Anderson \& Jap, 2005, 77). This does indeed appear to be an unambiguous example of opportunism-with-guile. However, earlier in the same paper the authors observe that the car company buyer used its power over the supplier in an overbearing manner (p. 77) and conclude that: 'Lest one be tempted to condemn the parts maker, recall the many stories in the business press of how some automakers have abused the trust and investment of their suppliers. Opportunism can run both ways.' (ibid). Thus, this case may well be another example of apparently opportunistic behaviour actually resulting from a self-defence reaction to prevent the breach of a conflicting principle. If so, then once more, it did not constitute a supplier-based threat to the buyer's interests, and hence did not require significant management attention to control the supplier's behaviour.

Mitrega \& Zolkiewski (2012) claim to identify opportunism on the part of an Internet infrastructure provider in a case: "The first contract ended and we continued dealing with each other. After a while I got reliable information from my colleague from another company that ... is offering much better terms of contract to new customers. These customers had better quality and more additional services at the same price ... When we contacted .... they said that these are special terms for new customers and maybe we can have just $10 \%$ discount 
... Only when we discussed the possibility of not lengthening the contract did they finally agree to providing us the same terms". (p. 891). This behaviour would only constitute opportunism if there was a market norm for suppliers to tell buyers the prices paid by all other customers. No such norm exists in most markets, and consequently this more closely resembles an example of competitive negotiating practice. The authors also suggest that the 'Worsening of negotiation position...' and a supplier becoming 'less oriented to my individual situation and more at procedures and documentation" (p. 890) are examples of passive opportunism - that is the: '...shirking or evasion of obligations in the ongoing relationship.' Or '...one of the parties to the exchange ...purposely withholding effort...' (Wathne \& Heide, 2000, 38). However, precisely the same supplier behaviours could have been motivated by a variety of factors other than opportunism, such as staffing problems, incompetence, changes in strategic objectives, unexpected demands from other strategically critical customers and so on. An unequivocal identification of opportunism is thus impossible.

On the other hand, the same authors describe an apparently clear example of Overtopportunism committed by a coal supplier who: '...used blackmail in terms of not supplying coal at the contracted price.' (Mitrega \& Zolkiewski, 2012, 890). One other case that may be an example of Overt-opportunism can be found in Wathne \& Heide (2000) who cite Klein (1996) and the relationship between General Motors and a supplier in 1926 described in Klein et al. (1978). A close reading of that original study reveals a supplier extracting as much profit as possible within the terms of an agreed contract. Since there is no mention of cheating, lying, the deliberate distortion of information or treachery of any kind this may be a case of Overt-opportunism possibly violating the norm of distributive justice. 
Finally, Cadden et al. (2015) show that transactional mechanisms at a strategic level led to opportunistic behaviour. A buying organisations used price renegotiation (by threatening with the use of substitute from alternative suppliers) when buying volumes increased and suppliers reciprocated this behaviour by trying to alter contract and relationship terms when buyer volumes decreased. 


\section{Reviewer comments and responses:}

Opportunism in buyer-supplier exchange: a critical examination of the concept and its implications for theory and practice.

We would like to thank the reviewers, who have taken a considerable amount of time to read and then provide a set of detailed and insightful comments on our revised submission. We have incorporated these and feel that they have helped us to further improve our paper. Please find our responses to reviewers in the table below and we have made any changes to the paper itself in red, as per the journal requirements. In addition, we have gone through the publisher layout guide and, in accordance with this document, the main body of the work is now fully in Times New Roman, 12 point and double-spaced and the headings/sub-headings are in alignment.

\begin{tabular}{|c|c|}
\hline Comments & Response \\
\hline $\begin{array}{l}\text { I would recommend that you add 'guile' to the } \\
\text { keywords to emphasise that this is where your } \\
\text { contribution lies. }\end{array}$ & 'Guile' has been added to the keywords. \\
\hline $\begin{array}{l}\text { I am not convinced that switching to the first } \\
\text { person at the end of the first paragraph in the } \\
\text { introduction works. I feel that explanation would } \\
\text { be better in the third person. }\end{array}$ & $\begin{array}{l}\text { This part of the Introduction has now been } \\
\text { changed into the third person. }\end{array}$ \\
\hline $\begin{array}{l}\text { I am also not convinced that the text "and our } \\
\text { main argument would be lost by including other } \\
\text { theoretical explanations" is needed here. It } \\
\text { seems out of place, you should always be able } \\
\text { to make your argument with clarity. }\end{array}$ & $\begin{array}{l}\text { This text has now been deleted and the } \\
\text { sentence has been amended to show a more } \\
\text { direct focus on our specific contribution based } \\
\text { on the concepts of guile. }\end{array}$ \\
\hline $\begin{array}{l}\text { Throughout I would expect you to always cite } \\
\text { the year when you cite an author's name, there } \\
\text { are numerous places where the year is not cited } \\
\text { for Williamson, Wathne \& Heide and Macneil, } \\
\text { for example. }\end{array}$ & This has been corrected throughout the article. \\
\hline $\begin{array}{l}\text { On page } 3 \text { you state "Through an evaluation of } \\
\text { direct and indirect evidence this paper argues } \\
\text { that Williamson's original definition of } \\
\text { opportunism with guile is not as common as } \\
\text { academic literature purports it to be." I am } \\
\text { rather confused by this statement, would this be } \\
\text { better phrased "Enactment of Williamson's } \\
\text { (year) definition....." }\end{array}$ & $\begin{array}{l}\text { We have changed this sentence accordingly and } \\
\text { it now reads much more clearly. }\end{array}$ \\
\hline Pg. 13, line 56 'paper' should be 'papers'. & We have made this change. \\
\hline $\begin{array}{l}\text { You need to check all the cross-references to } \\
\text { the tables, a number are incorrect. }\end{array}$ & $\begin{array}{l}\text { All the tables are now accurately cross- } \\
\text { referenced throughout the paper. }\end{array}$ \\
\hline $\begin{array}{l}\text { A cross-reference to the appropriate tables } \\
\text { would be useful at the beginning of section } 3.3 \\
\text { and } 4.1\end{array}$ & $\begin{array}{l}\text { Additional text has been added to the start of } \\
\text { these sections to highlight the specific tables } \\
\text { being referred to. In addition, similar insertions } \\
\text { have been made at other relevant points of the } \\
\text { paper. }\end{array}$ \\
\hline Pg. 28, lines 16/17 'includes' should be 'include'. & We have made this change. \\
\hline $\begin{array}{l}\text { Section } 4.2 \text { remains long-winded and difficult to } \\
\text { read through. Would it not be possible to add }\end{array}$ & $\begin{array}{l}\text { We have reviewed this section thoroughly and } \\
\text { agree that it is long-winded. Therefore, we }\end{array}$ \\
\hline
\end{tabular}




\begin{tabular}{|l|l|}
\hline $\begin{array}{l}\text { some of this material to Table 5, then a more } \\
\text { succinct discussion provided. }\end{array}$ & $\begin{array}{l}\text { have merged small parts of the second } \\
\text { paragraph with the first as these were } \\
\text { effectively dealing with the same point. Overall, } \\
\text { in this section we have now adopted shorter } \\
\text { paragraphs to make it more succinct and easier } \\
\text { to follow and have also removed any } \\
\text { extraneous aspects of the paragraphs } \\
\text { themselves. }\end{array}$ \\
\hline $\begin{array}{l}\text { While the new subheadings in the discussion } \\
\text { help with the structure, this key section remains } \\
\text { rather long and unwieldy. Please try to make this } \\
\text { discussion much more 'punchy'. }\end{array}$ & $\begin{array}{l}\text { We have removed a paragraph from this } \\
\text { section, which did cover a number of additional } \\
\text { examples and on reviewing this we felt it was } \\
\text { not adding anything significant to the } \\
\text { argument. In addition, we have also created a } \\
\text { separate conclusions and contributions section } \\
\text { that provides a clearer focus on the research } \\
\text { questions and makes the preceding section } \\
\text { shorter and less unwieldly. }\end{array}$ \\
\hline $\begin{array}{l}\text { You need to add more supporting citations for } \\
\text { the counter points you raise throughout the } \\
\text { paper, but especially on pg. } 31 \text { lines 12-19. }\end{array}$ & $\begin{array}{l}\text { As part of our review of section 4.2 (as per } \\
\text { above), this specific section has now been } \\
\text { integrated into the section 4.4 (summary of } \\
\text { discussion) and we have now provided } \\
\text { supporting citations, primarily linking the } \\
\text { argument to Kraljic's portfolio approach and } \\
\text { the rise in outsourcing. We hope these more } \\
\text { fully underpin the points we are making. }\end{array}$ \\
\hline
\end{tabular}


Tables and Figures

\begin{tabular}{|l|l|l|}
\hline Concept & Behaviour & Illustrative example \\
\hline Overt opportunism & $\begin{array}{l}\text { The open pursuit of } \\
\text { advantage or benefit by one } \\
\text { party to an exchange at the } \\
\text { expense of their trading } \\
\text { opposite(s). }\end{array}$ & $\begin{array}{l}\text { The supplier forces a price } \\
\text { increase through without } \\
\text { offering any justification. }\end{array}$ \\
\hline $\begin{array}{l}\text { Opportunism-with- } \\
\text { guile }\end{array}$ & $\begin{array}{l}\text { The hidden pursuit of } \\
\text { advantage or benefit, with } \\
\text { the deliberate intent to } \\
\text { deceive, by one party to an } \\
\text { exchange at the expense of } \\
\text { their trading opposite(s). }\end{array}$ & $\begin{array}{l}\text { The supplier requests a } \\
\text { deliberately distorted data } \\
\text { in justification, and hides } \\
\text { the distortion. }\end{array}$ \\
\hline
\end{tabular}

Table 1 - Example of the overt and with-guile forms of opportunism (source - authors)

\section{Operations Management}

Journal of Operations Management, International Journal of Operations and Production Management, Production, Planning and Control, Journal of Purchasing and Supply Management, Supply Chain Management an International Journal, Journal of Supply Chain Management, Journal of Production and Operations Management, Journal of Production Economics, International Journal of Physical Distribution and Logistics Management, Journal of Business logistics, International Journal of Logistics Management, Decision Sciences, European Journal of Operational Research

\section{Marketing}

Journal of Marketing Research, Journal of International Marketing, Psychology and Marketing, Journal of Business and Industrial Marketing, Journal of Retailing, Marketing Letters, Marketing Science, International Journal of Research in Marketing, Journal of the Academy of Marketing Science, Industrial Marketing Management, Journal of Marketing, European Journal of Marketing

\section{Other managerial areas}

Strategic Management Journal, Academy of Management Journal, Administrative Science Quarterly, British Journal of Management, Journal of Management

Table 2 - Relevant journals referring to the concept of opportunism 


\begin{tabular}{|c|c|c|c|}
\hline Citation & Context & $\begin{array}{l}\text { Opportunistic } \\
\text { Behaviour }\end{array}$ & Alternative Explanation \\
\hline $\begin{array}{l}\text { Cox \& } \\
\text { Thompson } \\
(1997\end{array}$ & $\begin{array}{l}\text { Construction } \\
\text { industry }\end{array}$ & $\begin{array}{l}\text { Adversarial } \\
\text { relationships } \\
\text { encourage } \\
\text { opportunism: } \\
\text { Increasing annual } \\
\text { turnover through } \\
\text { contractual claims }\end{array}$ & $\begin{array}{l}\text { Many reasons why } \\
\text { construction companies } \\
\text { present contractual claims } \\
\text { such as dealing with poor } \\
\text { management; inaccurate } \\
\text { forecasts; inaccurate } \\
\text { information. }\end{array}$ \\
\hline $\begin{array}{l}\text { Anderson } \\
\text { et al. } \\
(2000)\end{array}$ & $\begin{array}{l}\text { US car firms and die } \\
\text { manufacturers }\end{array}$ & $\begin{array}{l}\text { No evidence of buyer } \\
\text { opportunism, but } \\
\text { evidence of suppliers } \\
\text { causing delays that } \\
\text { could have been } \\
\text { attributed to } \\
\text { opportunism }\end{array}$ & $\begin{array}{l}\text { External suppliers do } \\
\text { more work to perfect the } \\
\text { dies than internal } \\
\text { suppliers. This takes } \\
\text { longer, hence delays. }\end{array}$ \\
\hline $\begin{array}{l}\text { Wathne } \\
\text { and Heide } \\
(2000)\end{array}$ & $\begin{array}{l}\text { Ford and supplier } \\
\text { Lear Corp. }\end{array}$ & $\begin{array}{l}\text { Supplier failed to } \\
\text { honour promises. }\end{array}$ & $\begin{array}{l}\text { Fords explanation of the } \\
\text { supplier's behaviour was } \\
\text { that rather than displaying } \\
\text { opportunism, Lear was } \\
\text { suffering from poor } \\
\text { management. }\end{array}$ \\
\hline $\begin{array}{l}\text { Anderson } \\
\text { and Jap } \\
(2005)\end{array}$ & $\begin{array}{l}\text { Car firm and Parts } \\
\text { supplier. }\end{array}$ & $\begin{array}{l}\text { Supplier reduces the } \\
\text { number of coats of } \\
\text { paint to cut costs of } \\
\text { unit price. }\end{array}$ & $\begin{array}{l}\text { Evidence of Buyer using } \\
\text { power over supplier. } \\
\text { Apparently opportunistic } \\
\text { behaviour is a result of } \\
\text { self-defence. }\end{array}$ \\
\hline $\begin{array}{l}\text { Mitrega } \\
\text { and } \\
\text { Zolkiewski } \\
(2012)\end{array}$ & $\begin{array}{l}\text { Internet } \\
\text { infrastructure } \\
\text { provider }\end{array}$ & $\begin{array}{l}\text { Offering better terms, } \\
\text { quality and additional } \\
\text { services to new } \\
\text { customers at the same } \\
\text { price as the long term } \\
\text { customer. }\end{array}$ & $\begin{array}{l}\text { There is no market norm } \\
\text { for suppliers to tell buyers } \\
\text { the prices paid by all } \\
\text { other customers. }\end{array}$ \\
\hline $\begin{array}{l}\text { Mitrega } \\
\text { and } \\
\text { Zolkiewski } \\
(2012)\end{array}$ & Coal supplier & $\begin{array}{l}\text { Coal supplier not } \\
\text { supplying coal at the } \\
\text { contracted price }\end{array}$ & $\begin{array}{l}\text { Close reading of the } \\
\text { contract showed that the } \\
\text { supplier was operating } \\
\text { within the terms of the } \\
\text { contractual agreement. } \\
\text { There was no cheating or } \\
\text { deliberate distortion of } \\
\text { information. }\end{array}$ \\
\hline $\begin{array}{l}\text { Cadden et } \\
\text { al. }(2015)\end{array}$ & Telecommunications & $\begin{array}{l}\text { The buying firm } \\
\text { threatened to use a } \\
\text { substitute from an } \\
\text { alternative supplier }\end{array}$ & $\begin{array}{l}\text { Traditional mechanism } \\
\text { tactics in renegotiations }\end{array}$ \\
\hline
\end{tabular}

Table 3 - Summary of direct evidence cases 


\begin{tabular}{|c|c|c|c|c|c|c|c|}
\hline Source & Context & $\begin{array}{l}\text { Respond } \\
\text { ents }\end{array}$ & $\begin{array}{l}\text { Opp. mean } \\
\text { (Likert scale } \\
\text { format) }\end{array}$ & $\begin{array}{l}\text { Mean } \\
\text { at or } \\
\text { below } \\
\text { mid- } \\
\text { point } \\
\text { of scale } \\
\text { (freq.) }\end{array}$ & $\begin{array}{l}\text { Rank of } \\
\text { opp. mean } \\
\text { in study }\end{array}$ & $\begin{array}{l}\text { Opp. as } \\
\text { dependent } \\
\text { variable }\end{array}$ & $\begin{array}{l}\text { Self- } \\
\text { report } \\
\text { or } \\
\text { others }\end{array}$ \\
\hline & & & $\underline{\text { Supplier }}$ Opportunism & & & & \\
\hline $\begin{array}{l}\text { Handley } \\
\& \\
\text { Benton, } \\
2012\end{array}$ & $\begin{array}{l}\text { Large US } \\
\text { based } \\
\text { companies } \\
\text { with } \\
\text { domestic and } \\
\text { /or offshore } \\
\text { business } \\
\text { processes. }\end{array}$ & Suppliers & $\begin{array}{l}1.43,1.40, \\
1.33,1.75, \\
1.51,1.50, \\
1.29,1.26, \\
1.43 \\
\text { (7 point) }\end{array}$ & 9 & Lowest & $\mathrm{Y}$ & Self \\
\hline $\begin{array}{l}\text { Lui \& } \\
\text { Ngo, } \\
2012\end{array}$ & $\begin{array}{l}\text { Trading } \\
\text { companies in } \\
\text { garment and } \\
\text { toy industries } \\
\text { in Hong } \\
\text { Kong and } \\
\text { suppliers in } \\
\text { China. }\end{array}$ & Buyers & $\begin{array}{l}2.8 \\
\text { (5 point) }\end{array}$ & 1 & Lowest & $\mathrm{N}$ & Others \\
\hline $\begin{array}{l}\text { Yang et } \\
\text { al. } 2011\end{array}$ & $\begin{array}{l}\text { Manufacturin } \\
\mathrm{g} \text { firms } \\
\text { covering a } \\
\text { wide range of } \\
\text { industries, } \\
\text { electronics, } \\
\text { computer } \\
\text { equipment, } \\
\text { chemicals, } \\
\text { apparel, } \\
\text { furniture, } \\
\text { food and } \\
\text { textiles in } \\
\text { China. }\end{array}$ & Buyers & $\begin{array}{l}2.46,3.62 \\
\text { ( } 7 \text { point) }\end{array}$ & & Lowest & $\mathrm{N}$ & Others \\
\hline $\begin{array}{l}\text { Yen \& } \\
\text { Barnes, } \\
2011\end{array}$ & $\begin{array}{l}\text { Anglo - } \\
\text { Taiwanese } \\
\text { buyer - seller } \\
\text { firms. }\end{array}$ & Buyers & $\begin{array}{l}3.07,2.96, \\
2.88,2.93 \\
\text { (7 point) }\end{array}$ & 4 & Lowest & $\mathrm{Y}$ & Others \\
\hline $\begin{array}{l}\text { Samaha } \\
\text { et al. } \\
2011\end{array}$ & $\begin{array}{l}\text { Fortune } 500 \\
\text { firm (seller) } \\
\text { and its } \\
\text { resellers }\end{array}$ & Buyers & $\begin{array}{l}2.23 \\
\text { (5 point) }\end{array}$ & 1 & $\begin{array}{l}\text { Second } \\
\text { lowest }\end{array}$ & $\mathrm{N}$ & Others \\
\hline
\end{tabular}




\begin{tabular}{|c|c|c|c|c|c|c|c|}
\hline & $\begin{array}{l}\text { (Channel } \\
\text { members). } \\
\text { Sectors } \\
\text { included } \\
\text { appliances, } \\
\text { automotive, } \\
\text { clothing, } \\
\text { electronics, } \\
\text { computers, } \\
\text { sport etc. }\end{array}$ & & & & & & \\
\hline $\begin{array}{l}\text { Barthéle } \\
\text { my, } 2011\end{array}$ & $\begin{array}{l}\text { French } \\
\text { franchise } \\
\text { chains. }\end{array}$ & Buyers & $\begin{array}{l}3.49 \\
\text { (7 point) }\end{array}$ & 1 & Lowest & $\mathrm{N}$ & Others \\
\hline $\begin{array}{l}\text { Barnes et } \\
\text { al. } 2010\end{array}$ & $\begin{array}{l}\text { Western } \\
\text { exporters } \\
\text { from US, } \\
\text { Canada, GB, } \\
\text { Ireland, } \\
\text { Australia and } \\
\text { New Zealand } \\
\text { and } \\
\text { importing } \\
\text { firms based } \\
\text { in Hong } \\
\text { Kong. }\end{array}$ & Buyers & $\begin{array}{l}2.96,3.16 \\
3.00,3.28 \\
\text { (7 point) }\end{array}$ & 4 & $\begin{array}{l}\text { Second } \\
\text { lowest }\end{array}$ & $\mathrm{Y}$ & Others \\
\hline $\begin{array}{l}\text { Ghosh \& } \\
\text { John, } \\
2009\end{array}$ & $\begin{array}{l}\text { US } \\
\text { engineering } \\
\text { intensive } \\
\text { industry } \\
\text { sectors. }\end{array}$ & Buyers & $\begin{array}{l}3.13 \\
\text { (7 point) }\end{array}$ & 1 & $\begin{array}{l}\text { Second } \\
\text { lowest }\end{array}$ & $\mathrm{N}$ & Others \\
\hline $\begin{array}{l}\text { Lui et al. } \\
2009\end{array}$ & $\begin{array}{l}\text { Hong Kong } \\
\text { trading firms } \\
\text { and Chinese } \\
\text { suppliers. }\end{array}$ & Buyers & $\begin{array}{l}2.9 \\
(5 \text { point })\end{array}$ & 1 & $\begin{array}{l}\text { Equal } \\
\text { lowest }\end{array}$ & $\mathrm{N}$ & Others \\
\hline $\begin{array}{l}\text { Lado et } \\
\text { al. } 2008\end{array}$ & $\begin{array}{l}\text { US catalogue } \\
\text { intermediarie } \\
\text { s affiliated } \\
\text { with a large } \\
\text { retail firm. }\end{array}$ & Buyers & $\begin{array}{l}2.24 \\
\text { (5 point) }\end{array}$ & 1 & Lowest & $\mathrm{N}$ & Others \\
\hline $\begin{array}{l}\text { Palmatie } \\
\text { r et al. } \\
2007\end{array}$ & $\begin{array}{l}\text { Business to } \\
\text { business } \\
\text { relationships } \\
\text { between a } \\
\text { major } \\
\text { Fortune } 500 \\
\text { company } \\
\text { (seller) and } \\
\text { its local } \\
\text { distributor } \\
\text { agents. } \\
\end{array}$ & Buyers & $\begin{array}{l}2.06,2.13 \\
(5 \text { point })\end{array}$ & 1 & $2^{\text {nd }}$ lowest & $\mathrm{N}$ & Others \\
\hline
\end{tabular}




\begin{tabular}{|c|c|c|c|c|c|c|c|}
\hline & $\begin{array}{l}\text { Businesses } \\
\text { cover } \\
\text { products } \\
\text { including } \\
\text { clothing, } \\
\text { hardware, } \\
\text { furniture and } \\
\text { appliances. }\end{array}$ & & & & & & \\
\hline $\begin{array}{l}\text { Heide et } \\
\text { al. } 2007\end{array}$ & $\begin{array}{l}\text { Business to } \\
\text { Business } \\
\text { relationships } \\
\text { between } \\
\text { manufacturer } \\
\text { s (suppliers) } \\
\text { of building } \\
\text { materials } \\
\text { (doors, } \\
\text { windows, } \\
\text { frames, stairs, } \\
\text { roofing } \\
\text { products) and } \\
\text { their } \\
\text { downstream } \\
\text { buyers. }\end{array}$ & Suppliers & $\begin{array}{l}1.38,1.56 \\
\text { ( } 7 \text { point })\end{array}$ & 2 & Lowest & $\mathrm{N}$ & Self \\
\hline $\begin{array}{l}\text { Morgan } \\
\text { et al. } \\
2007\end{array}$ & $\begin{array}{l}\text { UK } \\
\text { supermarket } \\
\text { retailers }\end{array}$ & Buyers & $\begin{array}{l}2.98 \\
\text { (7 point) }\end{array}$ & 1 & Lowest & $\mathrm{Y}$ & Others \\
\hline $\begin{array}{l}\text { Carson } \\
\text { et al. } \\
2006\end{array}$ & $\begin{array}{l}\text { Outsourced } \\
\text { R\&D } \\
\text { relationships } \\
\text { in new } \\
\text { product } \\
\text { development } \\
\text { in US }\end{array}$ & Buyers & $\begin{array}{l}\text { NB 13.81/28 } \\
\text { (responses } \\
\text { summed not } \\
\text { averaged) }\end{array}$ & 1 & Middle & $\mathrm{N}$ & Others \\
\hline $\begin{array}{l}\text { Wuyts \& } \\
\text { Geysken } \\
\text { s, } 2005\end{array}$ & $\begin{array}{l}\text { Small to } \\
\text { medium sized } \\
\text { firms in } \\
\text { Industrial and } \\
\text { commercial } \\
\text { machinery, } \\
\text { computer } \\
\text { equipment, } \\
\text { electronic, } \\
\text { electrical } \\
\text { equipment } \\
\text { and } \\
\text { components } \\
\text { in the } \\
\text { Netherlands. }\end{array}$ & Buyers & $\begin{array}{l}1.99 \\
\text { (7 point) }\end{array}$ & 1 & Lowest & $\mathrm{N}$ & Others \\
\hline Rokkan & Building & Buyers & 1.44 & 1 & Lowest & $\mathrm{Y}$ & Others \\
\hline
\end{tabular}




\begin{tabular}{|c|c|c|c|c|c|c|c|}
\hline $\begin{array}{l}\text { et al. } \\
2003\end{array}$ & $\begin{array}{l}\text { material } \\
\text { manufacturer } \\
\mathrm{s} \text { and } \\
\text { distributors. }\end{array}$ & & (7 point) & & & & \\
\hline $\begin{array}{l}\text { Skarmea } \\
\text { s et al. } \\
2002\end{array}$ & $\begin{array}{l}\text { Importing } \\
\text { distributors } \\
\text { purchasing } \\
\text { directly from } \\
\text { overseas. } \\
\text { Sectors: } \\
\text { textiles, pulp } \\
\text { and paper, } \\
\text { chemicals, } \\
\text { machinery } \\
\text { and electrical } \\
\text { machinery. }\end{array}$ & Buyers & $\begin{array}{l}2.63 \\
(5 \text { point })\end{array}$ & 1 & Lowest & $\mathrm{N}$ & Others \\
\hline $\begin{array}{l}\text { Dorsch } \\
\text { et al. } \\
1998\end{array}$ & $\begin{array}{l}\text { Random } \\
\text { sample of } \\
\text { purchasing } \\
\text { executives in } \\
\text { the US }\end{array}$ & Buyers & $\begin{array}{l}2.15,2.32, \\
3.05 \\
(6 \text { point })\end{array}$ & 3 & $\begin{array}{l}\text { Lowest- } 2^{\text {nd }} \\
\text { lowest }\end{array}$ & $\mathrm{N}$ & Self \\
\hline \multirow[t]{3}{*}{$\begin{array}{l}\text { Lee, } \\
1998\end{array}$} & $\begin{array}{l}\text { Australian } \\
\text { exporters and } \\
\text { foreign } \\
\text { exchange } \\
\text { partners. }\end{array}$ & Suppliers & $\begin{array}{l}2.1 \\
\text { (7 point) }\end{array}$ & 1 & Lowest & $\mathrm{Y}$ & Self \\
\hline & & & & & & & \\
\hline & & & $\begin{array}{l}\text { Buver } \\
\text { Opportunism }\end{array}$ & & & & \\
\hline $\begin{array}{l}\text { Kashyap } \\
\text { et al. } \\
2012\end{array}$ & $\begin{array}{l}\text { US } \\
\text { automotive } \\
\text { manufacturer } \\
\text { s and their } \\
\text { dealers. }\end{array}$ & Buyers & $\begin{array}{l}2.32 \\
\text { (7 point) }\end{array}$ & 1 & Lowest & $\mathrm{Y}$ & Self \\
\hline $\begin{array}{l}\text { Noordho } \\
\text { ff et al. } \\
2011\end{array}$ & $\begin{array}{l}\text { Dutch } \\
\text { business to } \\
\text { business } \\
\text { innovation } \\
\text { relationships. }\end{array}$ & Suppliers & $\begin{array}{l}2.84 \\
\text { (7 point) }\end{array}$ & 1 & Lowest & $\mathrm{N}$ & Others \\
\hline $\begin{array}{l}\text { Dev et } \\
\text { al. } 2011\end{array}$ & $\begin{array}{l}\text { Large hotel } \\
\text { firms in } \\
\text { North } \\
\text { America. }\end{array}$ & Buyers & $\begin{array}{l}1.99 \\
\text { (7 point) }\end{array}$ & 1 & Lowest & $\mathrm{Y}$ & Self \\
\hline $\begin{array}{l}\text { Ju et al. } \\
2011\end{array}$ & $\begin{array}{l}\text { Export } \\
\text { ventures in } \\
\text { China }\end{array}$ & Suppliers & $\begin{array}{l}2.42 \\
\text { (5 point) }\end{array}$ & 1 & Lowest & $\mathrm{Y}$ & Others \\
\hline $\begin{array}{l}\text { Liu et al. } \\
2010 b\end{array}$ & $\begin{array}{l}\text { Buyer- } \\
\text { supplier } \\
\text { relationships } \\
\text { in the context }\end{array}$ & Suppliers & $\begin{array}{l}3.25 \\
\text { (7 point) }\end{array}$ & 1 & $\begin{array}{l}\text { Second } \\
\text { Lowest }\end{array}$ & $\mathrm{Y}$ & Others \\
\hline
\end{tabular}




\begin{tabular}{|c|c|c|c|c|c|c|c|}
\hline & $\begin{array}{l}\text { of Chinese } \\
\text { household } \\
\text { appliances. }\end{array}$ & & & & & & \\
\hline $\begin{array}{l}\text { Wong et } \\
\text { al. } 2005\end{array}$ & $\begin{array}{l}\text { Manufacturer } \\
\text { s and } \\
\text { suppliers } \\
\text { various } \\
\text { industries in } \\
\text { Shanghai. }\end{array}$ & Suppliers & $\begin{array}{l}2.62 \\
(5 \text { point })\end{array}$ & 1 & $\begin{array}{l}\text { Second } \\
\text { Lowest }\end{array}$ & $\mathrm{Y}$ & Others \\
\hline $\begin{array}{l}\text { Cavusgil } \\
\text { et al. } \\
2004\end{array}$ & $\begin{array}{l}\text { US based } \\
\text { manufacturer } \\
\mathrm{s} \text { and } \\
\text { independent } \\
\text { foreign } \\
\text { distributers. }\end{array}$ & Suppliers & $\begin{array}{l}3.887,4.262, \\
4.543,4.585, \\
5.072 \\
\text { (7 point) }\end{array}$ & 1 & Mixed & $\mathrm{Y}$ & Others \\
\hline $\begin{array}{l}\text { Jap, } \\
2003\end{array}$ & $\begin{array}{l}\text { Supply base } \\
\text { of a major } \\
\text { firm in the } \\
\text { automotive } \\
\text { industry } \\
\text { involved in } \\
\text { on-line } \\
\text { reverse } \\
\text { auctions. }\end{array}$ & Suppliers & $\begin{array}{l}2.94 \\
\text { (7 point) }\end{array}$ & 1 & Lowest & $\mathrm{Y}$ & Others \\
\hline $\begin{array}{l}\text { Brown et } \\
\text { al. } 2000\end{array}$ & $\begin{array}{l}\text { US Hotel } \\
\text { Industry. }\end{array}$ & Buyers & $\begin{array}{l}2.232 \\
\text { (7 point) }\end{array}$ & 1 & Lowest & $\mathrm{Y}$ & Self \\
\hline $\begin{array}{l}\text { Dahlstro } \\
\text { m \& } \\
\text { Nygaard, } \\
1999\end{array}$ & $\begin{array}{l}\text { Norwegian } \\
\text { franchisee- } \\
\text { franchisor } \\
\text { relationships } \\
\text { in the oil } \\
\text { industry. }\end{array}$ & Buyers & $\begin{array}{l}3.06 \\
\text { (7 point) }\end{array}$ & 1 & Lowest & $\mathrm{N}$ & Others \\
\hline \multirow[t]{2}{*}{$\begin{array}{l}\text { Provan } \\
\& \\
\text { Skinner, } \\
1989\end{array}$} & $\begin{array}{l}\text { Farm and } \\
\text { Power } \\
\text { equipment } \\
\text { dealers and } \\
\text { primary } \\
\text { supplier } \\
\text { organisations } \\
\text { in US. } \\
\end{array}$ & Buyers & $\begin{array}{l}2.52 \\
\text { (6 point) }\end{array}$ & 1 & 2nd lowest & $\mathrm{Y}$ & Self \\
\hline & & & $\begin{array}{l}\text { Both Buyer } \\
\text { and Supplier } \\
\text { Opportunism }\end{array}$ & & & & \\
\hline $\begin{array}{l}\text { Caniëls } \\
\& \\
\text { Gelderm } \\
\text { an, } 2010\end{array}$ & $\begin{array}{l}\text { Information } \\
\text { and } \\
\text { communicati } \\
\text { on } \\
\text { Technology } \\
\text { professionals }\end{array}$ & $\begin{array}{l}\text { Buyers } \\
\text { and } \\
\text { suppliers }\end{array}$ & $\begin{array}{l}\text { Suppliers: } 2.54 \\
\text { Buyers: } 2.03 \\
\text { (5 point) }\end{array}$ & 2 & $\begin{array}{l}\text { Second } \\
\text { Lowest } \\
\text { Lowest }\end{array}$ & $\mathrm{Y}$ & Both \\
\hline
\end{tabular}




\begin{tabular}{|c|c|c|c|c|c|c|c|}
\hline & $\begin{array}{l}\text { in Dutch } \\
\text { local } \\
\text { government. }\end{array}$ & & & & & & \\
\hline $\begin{array}{l}\text { Liu et al. } \\
2010 \mathrm{a}\end{array}$ & $\begin{array}{l}\text { Distributors } \\
\text { and } \\
\text { Manufacturer } \\
\mathrm{s} \text { in Chinese } \\
\text { household } \\
\text { appliances. }\end{array}$ & $\begin{array}{l}\text { Buyers } \\
\text { and } \\
\text { suppliers }\end{array}$ & $\begin{array}{l}4.31 \\
\text { (7 point) }\end{array}$ & 0 & Lowest & $\mathrm{N}$ & Self \\
\hline $\begin{array}{l}\text { Liu et al. } \\
2009\end{array}$ & $\begin{array}{l}\text { Household } \\
\text { appliance } \\
\text { manufacturer } \\
\text { s and } \\
\text { distributors in } \\
\text { China. }\end{array}$ & $\begin{array}{l}\text { Buyers } \\
\text { and } \\
\text { suppliers }\end{array}$ & $\begin{array}{l}4.09 \\
\text { (7 point) }\end{array}$ & 0 & $\begin{array}{l}\text { Second } \\
\text { Lowest }\end{array}$ & $\mathrm{Y}$ & Others \\
\hline $\begin{array}{l}\text { Tokman } \\
\text { et al. } \\
2007\end{array}$ & $\begin{array}{l}\text { Greek SME, } \\
\text { 3PL } \\
\text { providers. }\end{array}$ & $\begin{array}{l}\text { Buyers } \\
\text { and } \\
\text { suppliers }\end{array}$ & $\begin{array}{l}3.08 \\
\text { (5 point) }\end{array}$ & 0 & $\begin{array}{l}\text { Second } \\
\text { lowest }\end{array}$ & $\mathrm{N}$ & Others \\
\hline $\begin{array}{l}\text { Kneyme } \\
\text { yer \& } \\
\text { Murphy, } \\
2005\end{array}$ & $\begin{array}{l}\text { Users and } \\
\text { Providers of } \\
\text { 3PL services } \\
\text { in US. }\end{array}$ & $\begin{array}{l}\text { Buyers } \\
\text { and } \\
\text { suppliers }\end{array}$ & $\begin{array}{l}\text { Suppliers: } 4.0 \\
\text { Buyers: } 3.1\end{array}$ & 1 & $\begin{array}{l}\text { Second and } \\
\text { third lowest }\end{array}$ & $\mathrm{N}$ & Others \\
\hline $\begin{array}{l}\text { Jap \& } \\
\text { Anderso } \\
n, 2003\end{array}$ & $\begin{array}{l}\text { Procurement } \\
\text { division of } 4 \\
\text { large } \\
\text { equipment } \\
\text { manufacturer } \\
\mathrm{s} \text { in } \\
\text { computing, } \\
\text { photography, } \\
\text { chemicals } \\
\text { and brewing. }\end{array}$ & $\begin{array}{l}\text { Buyers } \\
\text { and } \\
\text { suppliers }\end{array}$ & $\begin{array}{l}2.15 \\
\text { (7 point) }\end{array}$ & 1 & Lowest & $\mathrm{N}$ & Others \\
\hline $\begin{array}{l}\text { Jap, } \\
2001\end{array}$ & $\begin{array}{l}\text { Retailers and } \\
\text { manufactures } \\
\text { in chemical } \\
\text { industry. }\end{array}$ & $\begin{array}{l}\text { Buyers } \\
\text { and } \\
\text { suppliers }\end{array}$ & $\begin{array}{l}2.2 \\
\text { (7 point) }\end{array}$ & 1 & Lowest & $\mathrm{N}$ & Others \\
\hline
\end{tabular}

Table 4 - Empirical results: Opportunism in Buyer-Supplier Exchange

\begin{tabular}{|l|l|l|}
\hline Question & Question (principles being violated in bold) & $\begin{array}{l}\text { Frequency } \\
\text { with which the }\end{array}$ \\
\hline
\end{tabular}




\begin{tabular}{|c|c|c|}
\hline & & $\begin{array}{l}\text { question has } \\
\text { been used }\end{array}$ \\
\hline & The [trading opposite]: & \\
\hline & Honouring contracts/promises & \\
\hline 1 & sometimes promises to do things without actually doing them later. & 19 \\
\hline 2 & breaches formal or informal agreements to their benefit. & 15 \\
\hline 3 & does not always act in accordance with our contract(s). & 6 \\
\hline \multirow[t]{2}{*}{4} & $\begin{array}{l}\text { fails to deliver promises, as described in the contract, for its own } \\
\text { interests. }\end{array}$ & 1 \\
\hline & Truth Telling & \\
\hline 5 & alters the facts in order to get what they need. & 22 \\
\hline 6 & lies about certain things in order to protect its interests. & 19 \\
\hline 7 & exaggerates needs to get what they desire & 11 \\
\hline 8 & does not provide a completely truthful picture when negotiating & 9 \\
\hline 9 & has presented facts in such a way that has made them look good. & 6 \\
\hline 10 & $\begin{array}{l}\text { seems to believe that honesty does not pay when dealing with } \\
\text { partners }\end{array}$ & 4 \\
\hline 11 & makes false accusations & 3 \\
\hline \multirow[t]{2}{*}{12} & is candid with us (reverse scored) & 2 \\
\hline & Maintaining equity/distributive justice & \\
\hline 13 & $\begin{array}{l}\text { will try to take advantage of "holes" in our contract to further their } \\
\text { own interests. }\end{array}$ & 7 \\
\hline 14 & $\begin{array}{l}\text { feels it is OK to do anything within its means that will help further } \\
\text { its own interests. }\end{array}$ & 7 \\
\hline 15 & avoids fulfilling their responsibilities unless they are watched & 6 \\
\hline 16 & uses unexpected events to extract concessions from our firm. & 5 \\
\hline 17 & has benefited from our relationship to our detriment. & 4 \\
\hline 18 & withholds important information from us. & 4 \\
\hline 19 & fails to provide us with the support they are obliged to & 4 \\
\hline 20 & is unwilling to accept responsibility & 3 \\
\hline 21 & $\begin{array}{l}\text { expects my firm to pay for more than their fair share of the costs to } \\
\text { correct a problem }\end{array}$ & 2 \\
\hline 22 & $\begin{array}{l}\text { usually register a complaint if our company fails to meet our } \\
\text { cooperative agreements }\end{array}$ & 1 \\
\hline 23 & $\begin{array}{l}\text { expects to receive an unreasonably large share of the benefits from } \\
\text { our cooperative agreements }\end{array}$ & 1 \\
\hline 24 & tries to renegotiate contracts to its own advantage & 1 \\
\hline 25 & tends to escalate cost estimates as projects progress & 1 \\
\hline 26 & is less and less cooperative as projects progress & 1 \\
\hline 27 & $\begin{array}{l}\text { is reluctant to accept changes without receiving concessions and } \\
\text { compromises }\end{array}$ & 1 \\
\hline 28 & abuses displays of honesty on our part & 1 \\
\hline 29 & has coerced us unfairly in order to gain accessions & 1 \\
\hline 30 & is aloof toward us & 1 \\
\hline 31 & fails to provide proper notification & 1 \\
\hline
\end{tabular}




\begin{tabular}{|c|c|c|}
\hline & Observing bargaining norms & \\
\hline \multirow[t]{3}{*}{32} & does not negotiate from a good faith bargaining perspective & 6 \\
\hline & Avoiding shirking & \\
\hline & $\begin{array}{l}\text { If [the customer] were not able to detect it, how likely are members } \\
\text { of our organization to... }\end{array}$ & \\
\hline 33 & $\begin{array}{l}\text { not assign your best people to your business or account with [the } \\
\text { customer]? }\end{array}$ & 1 \\
\hline 34 & provide a lower than agreed to level of resources? & 1 \\
\hline 35 & withhold information that may be beneficial to [the customer]? & 1 \\
\hline 36 & not share the benefits of process improvements? & 1 \\
\hline 37 & delay making agreed to investments in employee training? & 1 \\
\hline \multirow[t]{3}{*}{38} & delay making agreed to investments in new technology? & 1 \\
\hline & Avoiding poaching & \\
\hline & $\begin{array}{l}\text { If [the customer] were not able to detect it, how likely are members } \\
\text { of our organization to... }\end{array}$ & \\
\hline 39 & $\begin{array}{l}\text { use potentially proprietary information obtained through your } \\
\text { relationship with [the customer] to gain favor with other clients? }\end{array}$ & 1 \\
\hline 40 & $\begin{array}{l}\text { use potentially proprietary information obtained through your } \\
\text { relationship with [the customer] to help win business with other } \\
\text { customers? }\end{array}$ & 1 \\
\hline \multirow[t]{2}{*}{41} & $\begin{array}{l}\text { use potentially proprietary information obtained through your } \\
\text { relationship with [the customer] to develop new services that you } \\
\text { can offer in the marketplace? }\end{array}$ & 1 \\
\hline & Avoiding deceit & \\
\hline 42 & has tried to deceive us on several occasions & 1 \\
\hline
\end{tabular}

Table 5 - Generic questions in empirical surveys 
Opportunism in buyer-supplier exchange: a critical examination of the concept and its implications for theory and practice

Stephen Kelly - Edge Hill University - stephen.kelly@edgehill.ac.uk (corresponding author)

Beverly Wagner - University of Strathclyde

John Ramsay - Staffordshire University 